\title{
Modelling rheumatoid arthritis: A hybrid modelling framework to describe pannus formation in a small joint
}

\author{
Fiona R Macfarlane ${ }^{1, *}$, Mark AJ Chaplain ${ }^{1}$, Raluca Eftimie ${ }^{2}$ \\ 1. School of Mathematics and Statistics, University of St Andrews, UK; \\ 2. Département de Mathématiques, University of Franche-Comté, Besançon, France;
}

\begin{abstract}
Rheumatoid arthritis (RA) is a chronic inflammatory disorder that causes pain, swelling and stiffness in the joints, and negatively impacts the life of affected patients. The disease does not have a cure yet, as there are still many aspects of this complex disorder that are not fully understood. While mathematical models can shed light on some of these aspects, to date there are not many such models that can be used to better understand the disease. As a first step in the mechanistic understanding of RA, in this study we introduce a new hybrid mathematical modelling framework that describes pannus formation in a small proximal interphalangeal (PIP) joint. We perform numerical simulations with this new model, to investigate the impact of different levels of immune cells (macrophages and fibroblasts) on the degradation of bone and cartilage. Since many model parameters are unknown and cannot be estimated due to a lack of experiments, we also perform a sensitivity analysis of model outputs to various model parameters (single parameters or combinations of parameters). Finally, we connect our numerical results with current treatments for RA, by discussing our numerical simulations in the context of various drug therapies using, for example, methotrexate, TNF-inhibitors or tocilizumab, which can impact different model parameters.
\end{abstract}

\section{Introduction}

Rheumatoid arthritis (RA) is a chronic inflammatory disorder that affects over $1 \%$ of the worldwide population [1]. The characteristics of RA include persistent inflammation of joints, which contributes to the degradation of cartilage, and damage to the bone(s) within the joint 2,33. Along with symptoms related to inflammation within joints, RA can increase the risk of other health issues such as cardiovascular events 3,4 , reduced cognitive function in the brain, fibrotic disease in the lungs, osteoporosis and a greater risk of cancers [5]. The main symptoms of RA include inflammation, pain, swelling and stiffness of joints, fatigue, weight loss. Generally, the smaller joints in the hands and feet are most likely to be affected [4], for example, proximal interphalangeal (PIP) joints, which are the middle joints on the non-thumb fingers of the hand, are commonly affected [6 8]. A key aspect of RA progression within a joint is the formation of a 'pannus' from the abnormally growing synovial membrane. The pannus is made up of mainly fibroblast-like synoviocytes (FLSs) and macrophage-like synoviocytes (MLSs). Through the production of inflammatory cytokines the proliferation, migration and cytokine secretion of these cell types increases leading to further inflammation. These cells also produce matrix degrading enzymes, like MMPs, which can breakdown cartilage and bone within a joint. In this work, we focus on these key features of pannus formation and growth and the subsequent cartilage and bone degradation. In the following subsection we provide further detail of these specific mechanisms. For more robust reviews of the biology of RA we direct the reader to the following papers 5,9 .

\subsection{Key biological background}

The synovial membrane is a soft connective tissue which lines synovial joints and allows for smooth movement through the secretion of lubricating synovial fluid [3,9]. This well vascularised tissue consists of a intimal layer of evenly dispersed cells and a sub-lining comprised of extracellular matrix interspersed with collagen fibrils and other matrix proteins [10]. The porous structure of the synovial membrane allows for the diffusion of nutrients, oxygen and chemokines into the joint [11. In a healthy joint, the intimal layer of the membrane is generally 12 cells thick and consists of fibroblast-like synoviocytes (FLSs) and macrophage-like synoviocytes (MLSs) evenly distributed and in equal amounts [10,11. Following the onset of RA, the synovial membrane expands through

*Corresponding author: frm3@st-andrews.ac.uk 
various inflammatory mechanisms, this growing membrane is known as a 'pannus' and can behave similarly to a locally invasive tumour spreading within the joint [10]. Through both an increase in the proliferation of FLSs and the infiltration of immune cells, such as bone-derived macrophages, the synovial membrane can expand to around 10-20 cells in thickness 5, 10, 12, 14. We focus on macrophages, or macrophage-like synoviocytes, and fibroblasts, or fibroblast-like synoviocytes, which form the majority of the pannus. However, the pannus can also consist of other immune cell types, including leukocytes, plasma cells, T cells and mast cells. Pannus formation and growth is controlled through the expression of pro-inflammatory cytokines by the cells, promoting further inflammation of the synovial membrane. The cytokines involved are vast and varied, including those with roles in immune cell recruitment, immune cell activation, chemotaxis of cells and degradation of the cartilage/bone. There can be a high level of heterogeneity in macrophage origin and function in the context of RA [14], as well as FLS phenotypes and cytokine profiles [15. In general, the pannus is heterogeneous consisting of diverse cellular and molecular signatures 10]. In recent years, distinct patterns have been recognised primarily according to composition, organisation and localisation of cellular infiltrates [10].

Cartilage is a connective tissue consisting of chondrocytes which produce a dense extra cellular matrix (ECM). Matrix metalloproteinases (MMPs) and tissue inhibitors of metalloproteinases (TIMPs) mediate cartilage destruction and are produced by B cells and FLSs in the RA setting $3,5,9,12$. Cells within the pannus can also stimulate cartilage degradation via direct cell contact mechanisms 3, 13. After cartilage has been damaged the bone underneath can become exposed. Bone erosion can be induced by cytokines that promote osteoclasts within the bone. In health, osteoclasts inhibit osteoblasts which produce new bone. In RA, the function of osteoclasts is increased, reducing the levels of new bone being formed leading to a reduction in bone formation [5, 9, 12 .

Once initiated the outcomes of RA cannot be reversed, however disease progression can be slowed and the symptoms of the disease can be reduced through a variety of treatment approaches. There are several classes of RA treatment drugs including non-steroidal anti-inflammatory drugs (NSAIDs), steroids and disease modifying antirheumatic drugs (DMARDs). These drugs can be used alone or in conjunction with other treatment approaches. Biological DMARDs are generally used in conjunction with conventional DMARDs, increasing their efficacy, and are considered to have a strong benefit-to-risk profile [16,17]. For a full description of the drugs used in rheumatoid arthritis treatment we refer the reader to the following papers [4,18,19]. We discuss some specific drugs and the effects they have in the RA context in Section 3.3 .

\subsection{Previous mathematical descriptions of RA}

Mathematical modelling is a useful tool to aid in the understanding of biological process at multiple spatial and temporal scales. We have recently reviewed previous mathematical models of rheumatoid arthritis, 20], and refer the reader to that paper for further detail. The few models existent in the literature, and described in our review paper, are mainly deterministic, however it has been suggested that accounting for stochasticity within RA may be key in understanding the evolution of the disease 9, 21 and predicting the success of RA treatments 22]. Furthermore, it has been found that there exists high levels of phenotypic and spatial heterogeneity of cells within the synovial membrane [23]. Understanding these single cell phenomena further has been highlighted as an important step in the identification of treatment targets 24 26]. Therefore in this study, we aim to develop a mathematical model that allows for stochastic effects within pannus formation to be taken into account. One approach to incorporate stochasticity within mathematical models is to use an individual-based (or agent-based) modelling approach 27.28]. This approach allows each cell to be described as an individual agent which follows a set of predetermined rules. This approach has been used in multiple areas of mathematical biology research including modelling the cellular immune response to viruses $[29$ and cancer [30]. These individual-based models can be computationally expensive especially when modelling a large number of agents. This expense can be reduced by using a hybrid modelling approach. For example, when modelling a chemical we may not necessarily be interested in modelling each individual molecule but the total local concentration instead. To model this a deterministic approach can be used, where the total local concentration is described rather than each molecule, this can be viewed as a tissue-level approach. To allow us to consider both the stochastic cellular level scale and deterministic tissue level dynamics of the system we can use a hybrid multiscale modelling approach 31. To model the deterministic components we can use classical methods such as non-spatial ordinary differential equations (ODEs) or spatial partial differential equations (PDEs). Hybrid approaches have been used to model various biological phenomena such as monocyte migration in the vasculature 32 , the cellular immune response to sepsis [33], inflammation in chronic obstructive pulmonary disease [34] or various mechanisms of cancer growth and development 35 38. In a similar way, in the context of rheumatoid arthritis, using multi-scale hybrid modelling approaches may be valuable in modelling disease progression and predicting the success of RA treatment. 


\subsection{Overview of paper}

In this work we develop a hybrid modelling framework to describe the growth and development of a pannus within a small PIP joint. We incorporate the dynamics of two cell populations, fibroblasts (or fibroblast-like synoviocytes) and macrophages (or macrophage-like synoviocytes) through a stochastic off-lattice individual-based modelling approach. The dynamics of cartilage and bone density, along with the evolution of MMPs are described via a deterministic discrete on-lattice approach. This work is a first-step to model the dynamics underlying RA progression. We are aware that several biological details are not included in this initial framework, however the methods used to implement each of the mechanisms described are those which could be used to include the necessary biological detail as the framework is improved upon. The paper is structured as follows. In Section 2, we describe the details of the framework and how each biological mechanism is described mathematically. In Section 3 , as an example we highlight some initial results of the framework and perform a sensitivity analysis to investigate the role of the parameters in the RA system. We additionally describe how the framework could be related to current rheumatoid arthritis treatments. Finally in Section 4 we discuss the key outputs of our framework, the requirements to validate the framework and the plans for developing the framework further.

\section{The discrete model}

In this section we introduce the hybrid modelling framework used to describe pannus formation in a small PIP joint. In the framework we consider 5 components: bone and cartilage densities, individual fibroblasts (or fibroblast-like synoviocytes) and macrophages (or macrophage-like synoviocytes) and the degrading proteases, matrix metalloproteinases (MMPs). We use an individual-based modelling approach to describe the dynamics of the cells, coupled with discretised PDEs to model cartilage density, bone density and MMP concentration. In Section 2.1, we describe the set-up of the initial spatial domain to replicate a PIP joint. The mathematical methods used to describe both fibroblast and macrophage dynamics are presented in Section 2.2. Section 2.3 contains the mechanisms used to describe the evolution of MMPs in the system. The methods used to describe both cartilage and bone density are given in Section 2.4. Finally, we describe how the on and off-lattice components of the model are coupled in Section 2.5 ,

\subsection{Set-up of the domain}

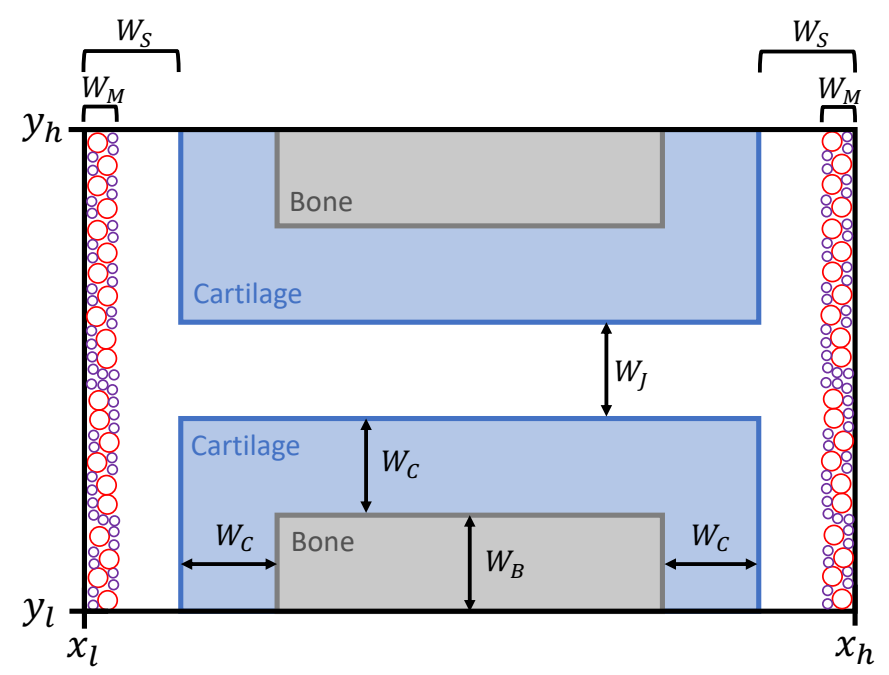

Figure 1: The initial spatial domain of the hybrid model. Shaded grey area represents the areas where bone is initially present, while shaded blue represents the areas where cartilage is initially present. The red and purple circles represent macrophages and fibroblasts, respectively. Note, that the initial position of both cell types of cells is randomised, so the positions here are just an example. The white space represents synovial fluid in which cells can freely move and proliferate, e.g., essentially free space in the system. Each of the parameters $W$ are shown on the diagram represent the length, height or width of each of the areas within the domain. Note, the diagram is not to scale. 
We consider a 2D spatial domain with $x \in\left[x_{l}, x_{h}\right]$ and $y \in\left[y_{l}, y_{h}\right]$. To simulate a small joint we consider the joint space to contain two bone ends, surrounded by cartilage with a small space between the two bones. We also consider the surrounding synovial membrane made up of macrophages and fibroblasts. A schematic diagram of this set-up is provided in Figure 1. To set-up the domain, we consider the width, length or height of each of these areas by defining the following parameters $W$, the descriptions of these parameters are available in Table 1 . Note, here as an initial formulation of the model we choose the bone and cartilage to be rectangular in shape, this could be updated to be more realistic (rounded) in future developments of the framework.

\begin{tabular}{|c|l|}
\hline Symbol & Description \\
\hline$W_{B}$ & the height of bone protruding into the domain \\
\hline$W_{C}$ & the width of the cartilage that surrounds the bone \\
\hline$W_{J}$ & the width of the joint space between the two bone ends \\
\hline$W_{M}$ & the initial width of the synovial membrane \\
\hline$W_{S}$ & the space between the cartilage and the edge of the domain \\
\hline
\end{tabular}

Table 1: Descriptions of the parameters used to set-up the initial spatial domain of the hybrid model as shown in Figure1.

\subsection{Cell dynamics}

Within the hybrid framework we describe two cell populations, fibroblasts and macrophages, using an individualbased approach. Each cell within both populations is tracked by the spatial position of the cell centre and the radius of the cell. We use an off-lattice modelling approach to describe the movement and proliferation of the cells to allow for a more realistic description of the cell dynamics in the RA joint. We track the total number of fibroblasts and macrophages over time, denoted by $N_{F}(t)$ and $N_{M}(t)$, respectively. We consider all cells to be spherical, with a homogenous radius that remains the same during birth and division processes. Each cell population has a uniform radius denoted as $R_{F}$ for the fibroblasts and $R_{M}$ for the macrophages. Initially, we randomly place $N_{F}(0)$ fibroblasts and $N_{M}(0)$ macrophages in the areas denoted by width $W_{M}$, as shown in Figure 1. For each cell a desired initial position is chosen then checked for overlap with previously placed cells, or the domain boundary as we consider a volume-exclusion process, whereby only one cell can occupy a particular area of space. If there is overlap, a new position is chosen for that cell and the process repeats, until all cells are placed. Both macrophages and fibroblasts can move via random motion, divide and die in our model. These processes are described in the following subsections. We note that each cell has the same potential to divide, die or migrate as all other cells within the same population. That is, we consider homogeneous populations of cells. Furthermore, we do not consider the influx or recruitment of immune cells into the domain, that is, all cells remain within the spatial domain from birth to death.

\subsubsection{Cell movement}

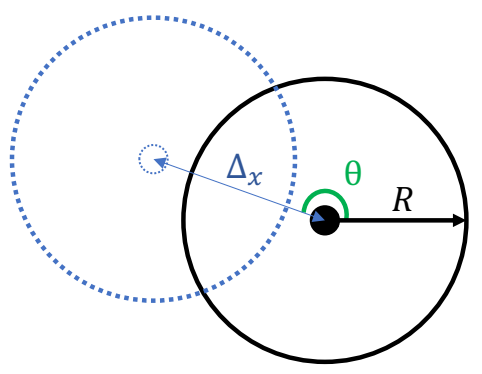

Figure 2: Schematic of the off-lattice cell movement mechanism. The solid black circle represents the initial cell position where the cell has radius $R$. A desired position is chosen by selecting a random angle $\theta \in[0,2 \pi]$ and then placing the cell centre at a distance of $\Delta_{x}$ from the original position in the direction $\theta$. The desired position (blue dashed circle) is chosen as the cell's updated position if no other cells, cartilage or bone overlap this spatial position. If this position does overlap the cell movement process is aborted.

At every time-step each cell can move randomly with probability, $\lambda_{F}$ or $\lambda_{M}$. If the cell is permitted to move, then a new direction is chosen from an angle $\theta$ between 0 and $2 \pi$ for the cell to move in, as shown in Figure 2 . The new 
position is calculated as a jump of length $\Delta_{x}=\Delta_{x c e l l s}$, to ensure proper scaling of movement probabilities. The movement to this desired position is only permitted if the new position is within the boundary, does not overlap with any other cells or with cartilage/bone density. If any of these things prevent movement, the movement jump is aborted.

\subsubsection{Cell population growth}

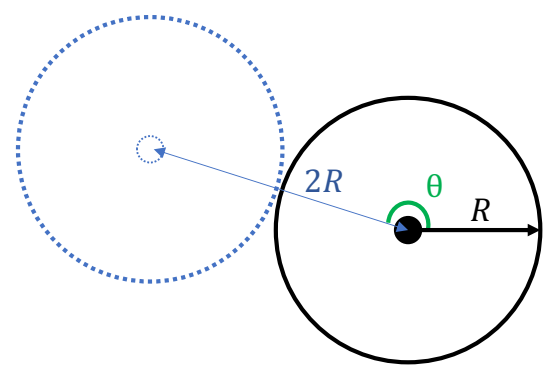

Figure 3: Schematic of the off-lattice cell division mechanism. The solid black circle represents the original cell position where the cell has radius $R$. The cell will divide into two identical daughter cells, one cell will remain on the original cell's position and the other will be placed at a desired position adjacent to the original cell's position. A desired position for the second daughter cell is chosen by selecting a random angle $\theta \in[0,2 \pi]$ and then placing the cell centre at a distance of $2 R$, i.e., the cell's diameter, from the original position in the direction $\theta$. The desired position (blue dashed circle) is chosen as the daughter cell's position if no other cells, cartilage or bone overlap this spatial position. If this position does overlap the cell division process is aborted and the original cell remains.

It has been shown that a major factor controlling cell division is a random event controlled by a transition probability [39]. Therefore, to incorporate cell division into our model we allow for this random event by allocating a probability of division at each time-step to each cell. This random event occurs once the cell has reached a minimum size, however for simplicity in this model we do not incorporate cell cycle or cell size, these details could be explicitly considered in future developments of the model. At every time-step, each cell has a probability of dying or dividing. The probability of dying is given by $\kappa_{F}$ or $\kappa_{M}$ while the probability of dividing is given by $\alpha_{F}$ or $\alpha_{M}$, for fibroblasts and macrophages respectively. If the cell is assigned to die at a given time-step, it is simply removed from the simulation instantly. If the cell is permitted to divide, then the cell splits into two identical daughter cells. One is placed on the parent cell's original position and the other is placed in a chosen position adjacent to this, as shown in Figure 3. This adjacent position is chosen from an angle $\theta$ between 0 and $2 \pi$. The new position is set at a length of twice the radius of the cell $R_{F}$ or $R_{M}$, i.e., the cell's diameter. This new cell is only placed on this position if the position is within the boundary, does not overlap with any other cells or with cartilage/bone density. If any of these things prevent division, the process is aborted and the parent cell remains in the original position.

\subsection{MMP dynamics}

We describe the dynamics of MMPs in the system as a deterministic process, as we are interested in the total local concentration of MMPs rather than each individual molecule. We formulate this as a partial differential equation (PDE) and then discretise this to allow for numerical simulations to be considered. Note, for this we have to assign a spatial grid or lattice for the MMPs to be contained within. We consider that MMPs can diffuse on the lattice and decay naturally over time. We also consider that MMPs are secreted by both fibroblasts and macrophages, and therefore we consider the density of cells to contribute to the local MMP concentration. Furthermore, we impose a local maximum restriction on the MMP concentration to ensure the local concentration is bounded. Mathematically these assumptions can be captured by the PDE,

$$
\frac{\partial C M M P}{\partial t}=\lambda_{C M M P} \nabla^{2} C M M P+\left(\beta_{F} \rho_{F}+\beta_{M} \rho_{M}\right)(1-C M M P)-\kappa_{C M M P} C M M P,
$$

where $C M M P=C M M P(\mathbf{x}, t)$ is the local concentration of MMPs at position $\mathbf{x}$ at time $t, \lambda_{C M M P}$ is the diffusivity of MMPs, $\beta_{F}$ and $\beta_{M}$ are the rates of production of MMPs by fibroblasts and macrophages, respectively, $\rho_{F}=\rho_{F}(\mathbf{x}, t)$ 
and $\rho_{M}=\rho_{M}(\mathbf{x}, t)$ are the cell densities of fibroblasts and macrophages, respectively, and $\kappa_{C M M P}$ is the decay rate of MMPs. The method used to discretise the PDE can be found in A. We solve the PDE with zero-Neumann boundary conditions to allow all MMPs to remain within the domain. We note that as MMPs are a small molecule, MMPs can enter the areas of cartilage and bone density freely and there are no spatial restrictions on the MMPs diffusion within the domain.

\subsection{Cartilage and Bone dynamics}

For the initial model we only consider local decay of cartilage and bone densities, we formulate these as,

$$
\begin{aligned}
& \frac{\partial \rho_{C}}{\partial t}=-\kappa_{\rho_{C}} \rho_{C} C M M P \\
& \frac{\partial \rho_{B}}{\partial t}=-\kappa_{\rho_{B}} \rho_{B} C M M P
\end{aligned}
$$

where $\rho_{C}=\rho_{C}(\mathbf{x}, t)$ and $\rho_{B}=\rho_{B}(\mathbf{x}, t)$ are the densities of cartilage and bone, respectively, and $\kappa_{\rho_{C}}$ and $\kappa_{\rho_{B}}$ are the rates of degradation of cartilage and bone by MMPs, $C M M P=C M M P(\mathbf{x}, t)$, respectively. The method used to discretise the Equations (2.2)-2.3 can be found in A. We solve these equations with zero-Neumann boundary conditions to ensure all components remain within the spatial domain.

\subsection{Linking the off-lattice components of the model with on-lattice components}

As described in the previous subsections the dynamics of the fibroblasts and macrophages are described using an offgrid or off-lattice approach, however the dynamics of the MMPs, cartilage and bone are constrained to a discretised grid on the spatial domain. Therefore, we have to couple these methods to allow interactions between the different components of the model.

\subsubsection{Secretion of MMPs}

For simplicity, we assume that cells produce MMPs at the centre position of the cell. To allow the concentration of MMPs to remain on our discretised grid, we calculate the nearest grid-position to the cell centre, and add the desired MMP concentration to this grid position.

\subsubsection{Volume-exclusion}

To ensure that the cells do not move or divide onto areas of the domain that contains bone or cartilage we need to evaluate the position of the whole cell on the grid. If no cartilage or bone degradation has occurred we simply check whether the new desired position is within $\pm R$ of the initial areas of the domain containing cartilage or bone, as depicted in Figure 1. That is, we check any part of the cells circumference will be within the initial cartilage or bone domain areas. If bone or cartilage degradation has occurred then we want to ensure that the cell can now move into this free space. To do this we find equally distributed points on each cell's circumference and then calculate the nearest grid-position of each of these points. We then check whether there is cartilage or bone present on those positions, to ensure the cell is able to move. Note, here we make the assumption that a cell can only inhabit an area of space if the cartilage and bone density is zero, however in future work we could allow cells to inhabit areas if these densities are below some threshold value, if appropriate.

\section{Numerical simulations and sensitivity analysis}

To visualise the framework results we can run numerical simulations of the model as described in the previous section. To perform numerical simulations of the hybrid framework we use MATLAB, the details of the explicit method used to solve the PDEs is given in $\mathrm{A}$. The parameters used in the initial runs of the simulations are given in Table 2 . Every iteration of the simulation is run with the same initial condition. A schematic overview of the key components, biological mechanisms and key parameters in the model is provided in Figure 4. 


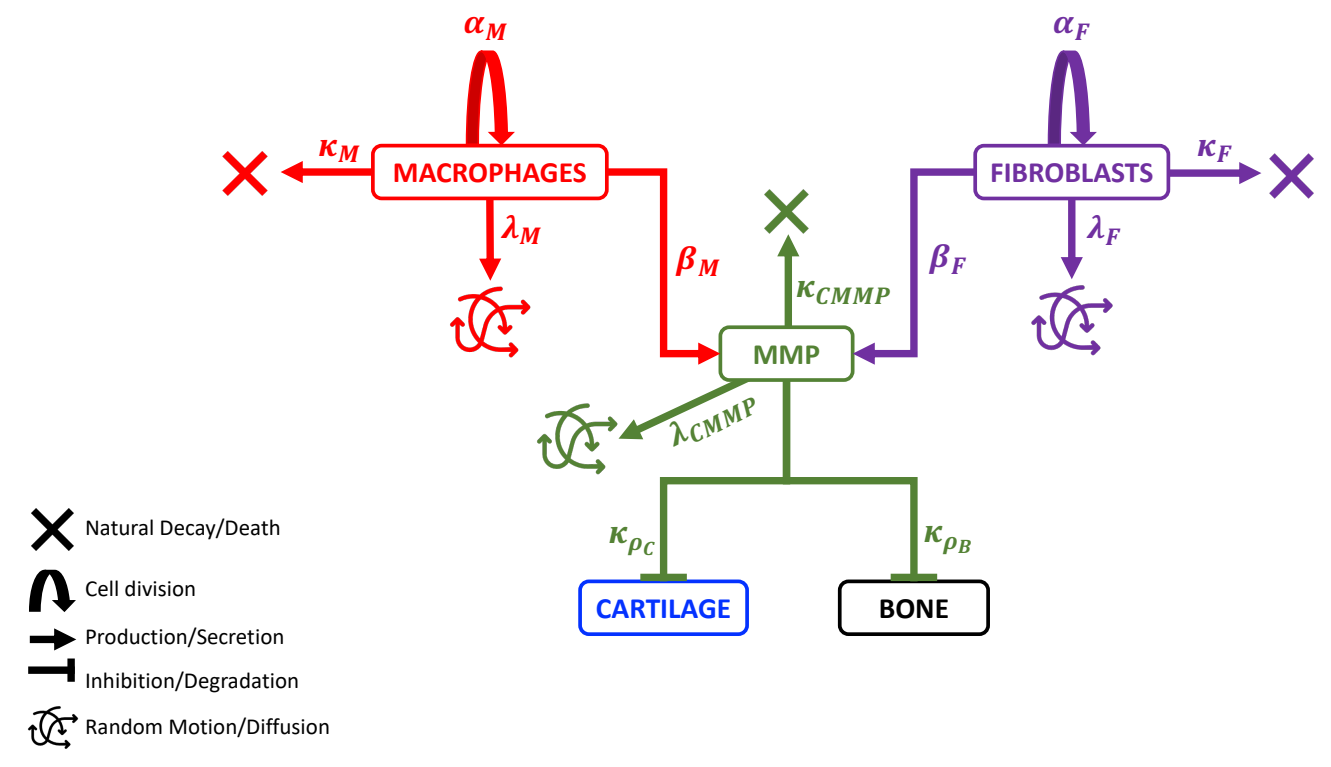

Figure 4: Schematic describing the components, mechanisms and parameters included in the hybrid model. Here, $\alpha_{F}$ and $\alpha_{M}$ represent the probability of cell division for fibroblasts and macrophages, respectively, while $\kappa_{F}$ and $\kappa_{M}$, represent the probability of cell death. The probability of cell movement for fibroblasts and macrophages is given by $\lambda_{F}$ and $\lambda_{M}$, respectively. The MMP secretion rates of fibroblasts and macrophages are given by $\beta_{F}$ and $\beta_{M}$, respectively. The MMPs decay naturally at the rate $\kappa_{C M M P}$ and can diffuse in the spatial domain at the rate $\lambda_{C M M P}$. The MMPs additionally degrade both cartilage and bone at the rates $\kappa_{\rho_{C}}$ and $\kappa_{\rho_{B}}$, respectively. 


\subsection{Increasing the division probabilities of macrophages and fibroblasts}
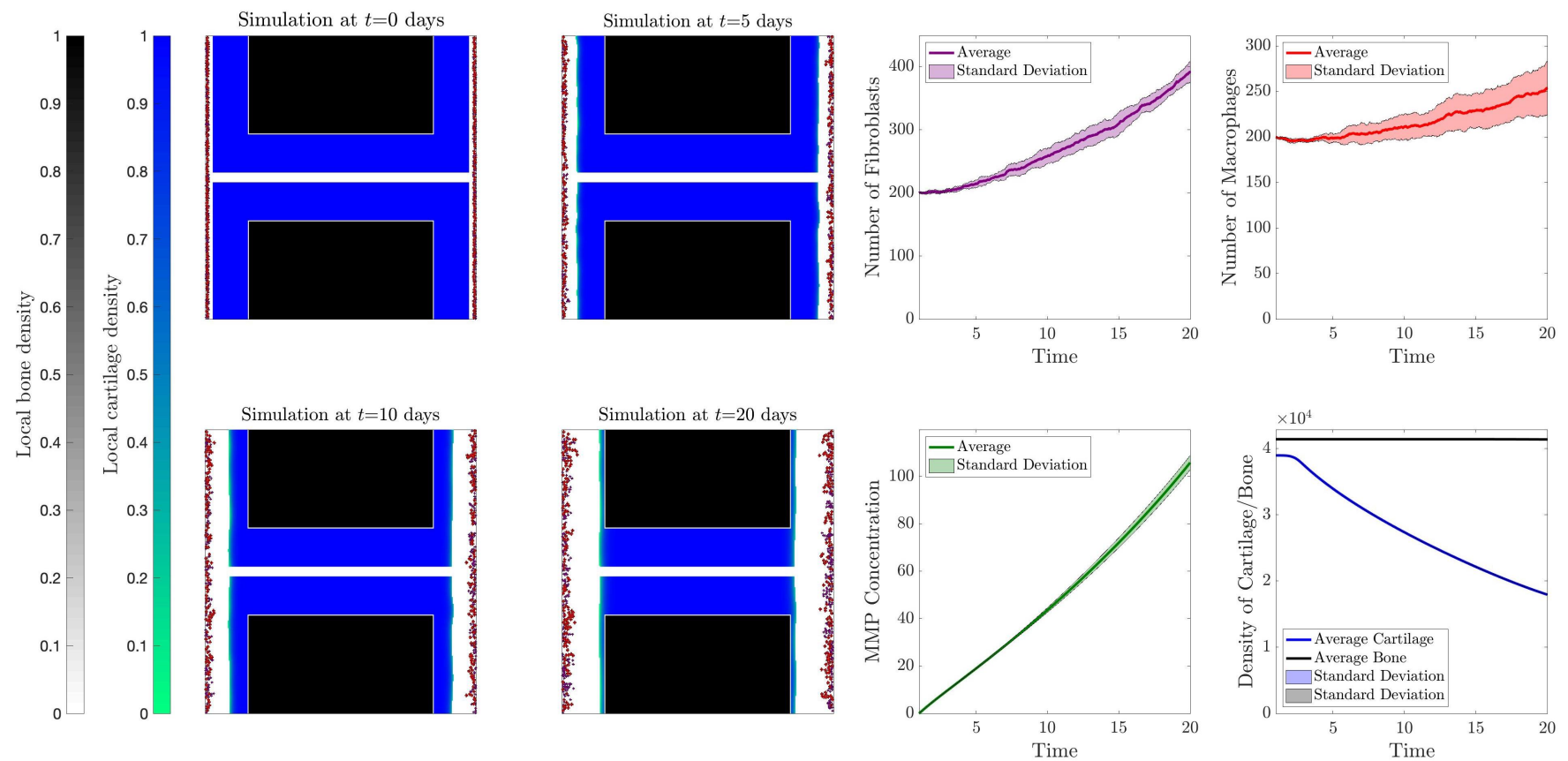

Figure 5: Example results where all parameters are those given in Table 2. Left-hand plots: Panels show the visualisation of spatial results of one of the runs of the simulation at the time-points $t=\{0,5,10,20\}$ days. The red dots are macrophages, the purple dots are fibroblasts, the blue-green surface is cartilage density and the black-grey surface is bone density. White space represents space in which cells can move freely in the joint, e.g., synovial fluid. Right-hand plots: The plots show the cell number, concentration or density over time averaged over 5 simulation runs with the standard deviation shaded. The number of fibroblasts is given in purple, the number of macrophages in red, the global MMP concentration in green, the global cartilage density in blue and the global bone density in black.

As an example of the simulation results of the model we consider the cases where all parameter values are taken to be the values given in Table 2 (the baseline case). The simulation results for the first 20 days are shown in Figure 5 . We plot the spatial distributions of cells, bone and cartilage at four time-points in the left-hand panels. We additionally plot the average number of fibroblasts, average number of macrophages, average global MMP concentration, average global cartilage density and average global bone density from 5 runs of the simulation in the right-hand plots. Here, global concentration/density refers to the sum of the MMP concentration or cartilage/bone densities across the whole spatial domain. We show the standard deviation between the 5 runs as the shaded area on each plot. We can see from these results that in this case, the cartilage density does decrease as MMP concentration increases, however no bone degradation occurs. In all of the line plots the standard deviation between runs is relatively low, especially in MMP concentration and the cartilage density plots. 

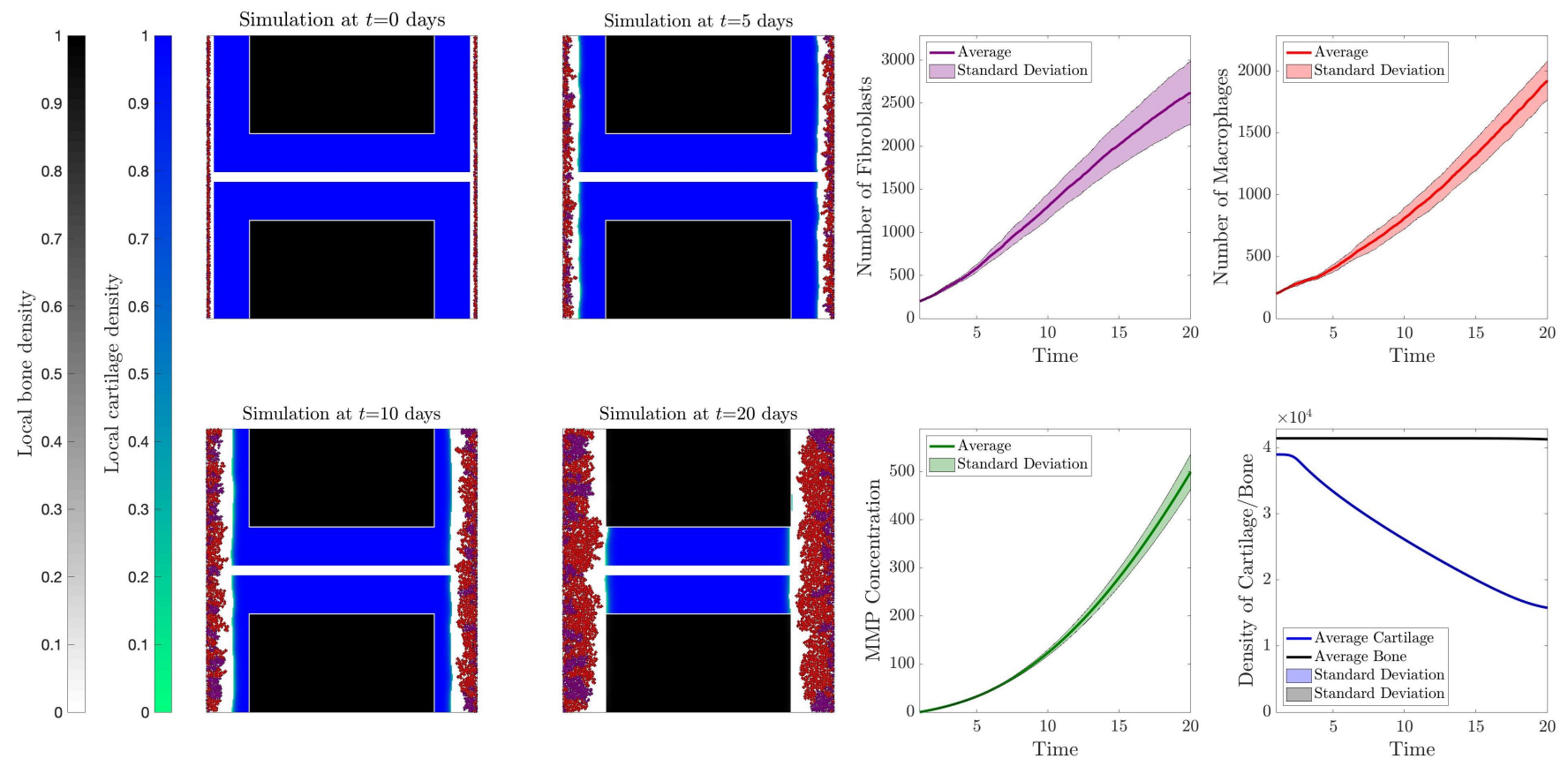

Figure 6: Example results where all parameters are those given in Table 2 except $\alpha_{F}$ and $\alpha_{M}$ which are ten times the given value. Left-hand plots: Panels show the visualisation of spatial results of one of the runs of the simulation at the time-points $t=\{0,5,10,20\}$ days. The red dots are macrophages, the purple dots are fibroblasts, the blue-green surface is cartilage density and the black-grey surface is bone density. White space represents space in which cells can move freely in the joint, e.g., synovial fluid. Right-hand plots: The plots show the cell number, concentration or density over time averaged over 5 simulation runs with the standard deviation shaded. The number of fibroblasts is given in purple, the number of macrophages in red, the global MMP concentration in green, the global cartilage density in blue and the global bone density in black.

We can also consider cases where we expect slightly more immune cells in the affected joint system, to replicate a case where immune activity has been amplified. As an example, in Figure 6 we show results of the case where immune cell proliferation is increased ten-fold. From these results, we observe a larger increase in the number of fibroblasts and macrophages over time as expected. We also see that the concentration of MMPs is increased in comparison to the case shown in Figure 5. In this case, the cartilage degradation is more severe than the previous case, however the bone density is retained. Once again, we observe low standard deviation between runs for all of the line plots. 

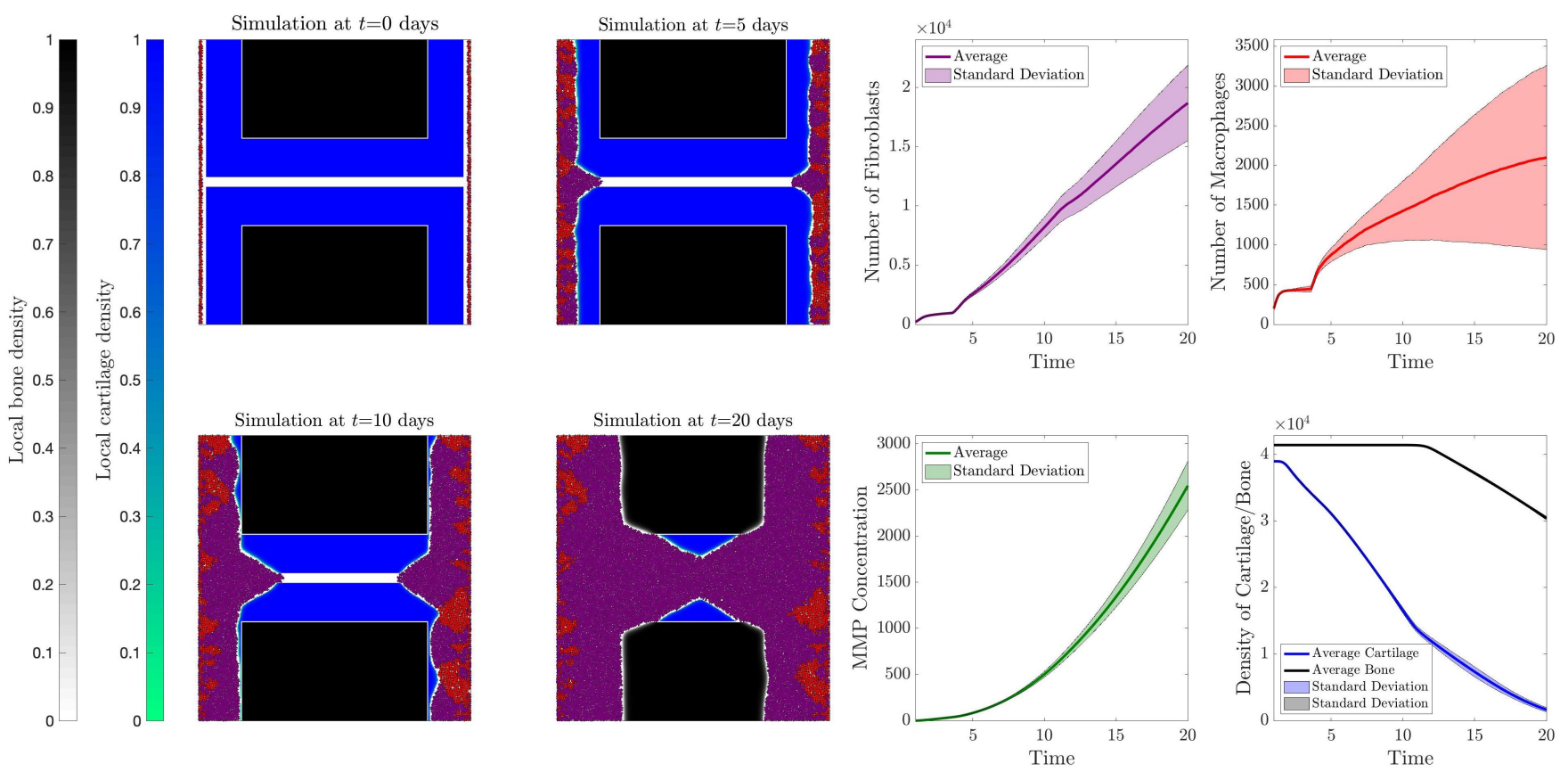

Figure 7: Example results where all parameters are those given in Table 2 except $\alpha_{F}$ and $\alpha_{M}$ which are one hundred times the given value. Left-hand plots: Panels show the visualisation of spatial results of one of the runs of the simulation at the time-points $t=\{0,5,10,20\}$ days. The red dots are macrophages, the purple dots are fibroblasts, the blue-green surface is cartilage density and the black-grey surface is bone density. White space represents space in which cells can move freely in the joint, e.g., synovial fluid. Right-hand plots: The plots show the cell number, concentration or density over time averaged over 5 simulation runs with the standard deviation shaded. The number of fibroblasts is given in purple, the number of macrophages in red, the global MMP concentration in green, the global cartilage density in blue and the global bone density in black.

Finally, we consider a case where we expect many more immune cells in the affected joint, to replicate an aggressive form of rheumatoid arthritis, where immune cell activity has been greatly amplified. As an example, we show results of the case where immune cell proliferation is increased one hundred-fold in Figure 7. Here, we observe a larger increase in the number of fibroblasts and macrophages over time as expected. We also see that the concentration of MMPs is increased in comparison to the previous cases. Furthermore, the cartilage degradation is more severe than the previous cases and we begin to see bone degradation occurring. Here, the standard deviation is much larger in the cell numbers. Interestingly though there is still very low standard deviation in the MMP concentration, and cartilage and bone densities. This suggests that even stochasticity in the number or spatial position of cells, we still expect similar levels of cartilage and bone degradation. We confirm this by plotting the final spatial distributions of each run of the simulation for the case shown in Figure 7, in Figure 8 , where we observe a variety of spatial patterns arising from the cell dynamics, which do not appear to affect the overall outputs of the model.
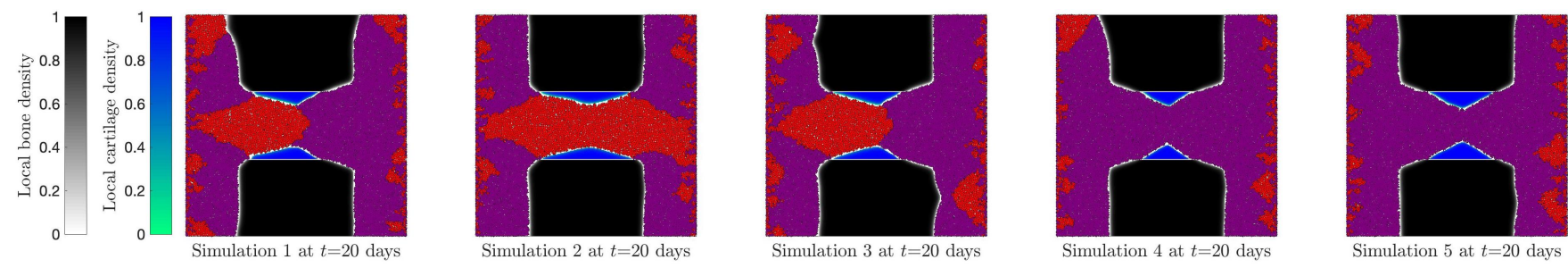

Figure 8: Panels show final visualisations of the 5 individual simulation runs to compare the spatial distributions of cells. The red dots are macrophages, the purple dots are fibroblasts, the blue-green surface is cartilage density and the black-grey surface is bone density. White space represents space in which cells can move freely in the joint, e.g., synovial fluid. Here, all parameters are base case except $\alpha_{F}$ and $\alpha_{M}$ which are one hundred times the base value. 


\subsection{Sensitivity analysis}

The initial parameter choices of our model are taken or estimated from a range of theoretical and experimental work, as described in $B$. We note that this parameter estimation can lead to parameter uncertainties, and therefore we utilise sensitivity analysis techniques to investigate how sensitive the outputs of the model are to changes in the parameter value inputs. These techniques can help identify the key parameters within the model and identify which parameters require more accurate values to ensure accuracy of the model output 40.41. For each sensitivity analysis we incorporate a vector $S$ to represent scalar values to multiply the parameter under investigation, where,

$$
S=[0.001,0.005,0.01,0.05,0.1,0.5,1,5,10,50,100]
$$

We use the notation $S_{n}=S(n)$ for $n=1, \ldots, 11$ to denote the value of each component. The maximum value of $S$ is chosen to ensure all probabilities are less than or equal to 1 . We then investigate parameters by setting,

$$
\Theta_{n}=S_{n} \Theta \quad \text { for } n=1, \ldots, 11
$$

where $\Theta$ is the parameter investigated. The model outputs that we focus on are the total number of fibroblasts and macrophages over time, along with the global MMP concentration, global cartilage density and global bone density over time. Where global concentration/density refers to the sum of the concentrations/densities across all spatial positions in the domain. Each parameter setting is run 5 times and the average is plotted along with the standard deviation of these runs.

\subsubsection{Sensitivity analysis of single characteristics}

We perform a local 'robustness' sensitivity analysis where one single input parameter is varied while all others are kept at fixed values. We keep all parameter values to be constant, choosing the values given in Table 2 and then testing scaled values of the parameter under investigation using the components of vector $S$, as defined in Equation (3.1) as our scaling values.
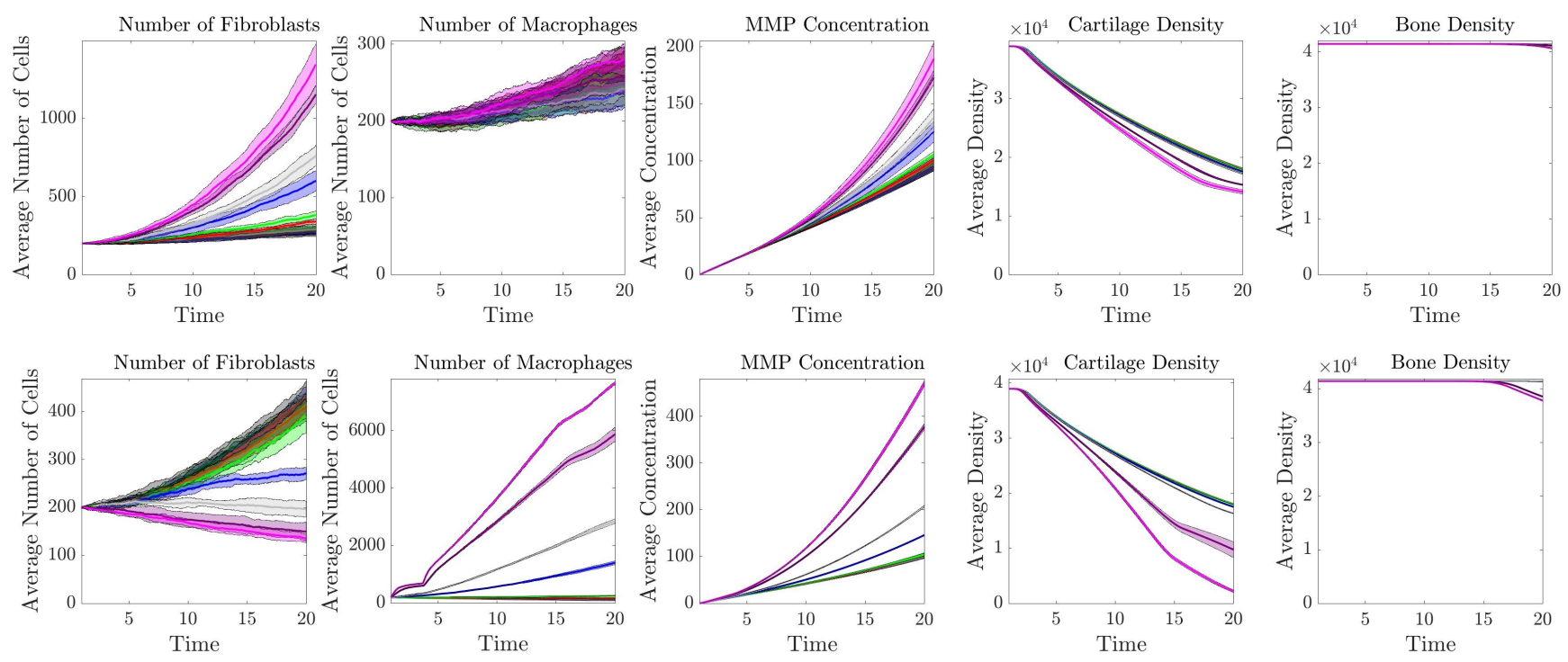

$$
-S_{1}=0.001-S_{2}=0.005-S_{3}=0.01-S_{4}=0.05-S_{5}=0.1-S_{6}=0.5-S_{7}=1-S_{8}=5-S_{9}=10-S_{10}=50-S_{11}=100
$$

Figure 9: Sensitivity analyses of the movement probability of fibroblasts $\lambda_{F}$, (top row), or the movement probability of macrophages $\lambda_{M}$, (bottom row). The five columns represent the five key outputs of the model: the total number of fibroblasts over time, the total number of macrophages over time, the global MMP concentration over time, the global cartilage density over time and the global bone density over time. In each subplot the results for each scalar value of $S_{n}$ is plotted as indicated by the legend, where the original values of $\lambda_{F}$ or $\lambda_{M}$ are multiplied by $S_{n}$ in each case. The solid line is the average of five runs of the simulation and the shaded area in the same colour is the standard deviation between runs. 
In Figure 9, we consider the movement probabilities of fibroblasts and macrophages, $\lambda_{F}$ and $\lambda_{M}$. In the case where we vary $\lambda_{F}$ only (top row), for $S_{n} \leq S_{7}=1$ there is no significant difference in all 5 outputs. However, for other values (i.e., $S_{n} \geq S_{8}=5$ ) there is a gradual increase in the number of fibroblasts and global MMP concentrations as $\lambda_{F}$ increases. For larger values of $S_{n} \geq S_{10}=50$ we additionally observe an increase in the degradation of cartilage, however for all $S_{n}$ there is no significant difference in total macrophage number or bone degradation levels. In the case where we vary $\lambda_{M}$ only (bottom row), for $S_{n} \leq S_{7}=1$ there is no significant difference in all 5 outputs. For the values of $S_{n} \geq S_{8}=5$ we observe an increase in the number of macrophages, an increase in global MMP concentration and a decrease in the number of fibroblasts in the system over time as we increase $\lambda_{M}$. Furthermore, for values $S_{n} \geq S_{10}=50$ we additionally observe an increase in both cartilage and bone degradation over time. In both cases, for varying $\lambda_{F}$ or $\lambda_{M}$, the standard deviation between runs is relatively low. These results suggest that although varying the movement probability of fibroblasts or macrophages may alter the immune cell numbers, varying these parameters does not significantly alter the cartilage and bone degradation unless large values are chosen. As each cell can only move and proliferate into free space around them increasing their movement probability allows them to find areas of free space quicker, which subsequently allows them to proliferate more freely resulting in higher numbers of cells permitted in the system, as observed.
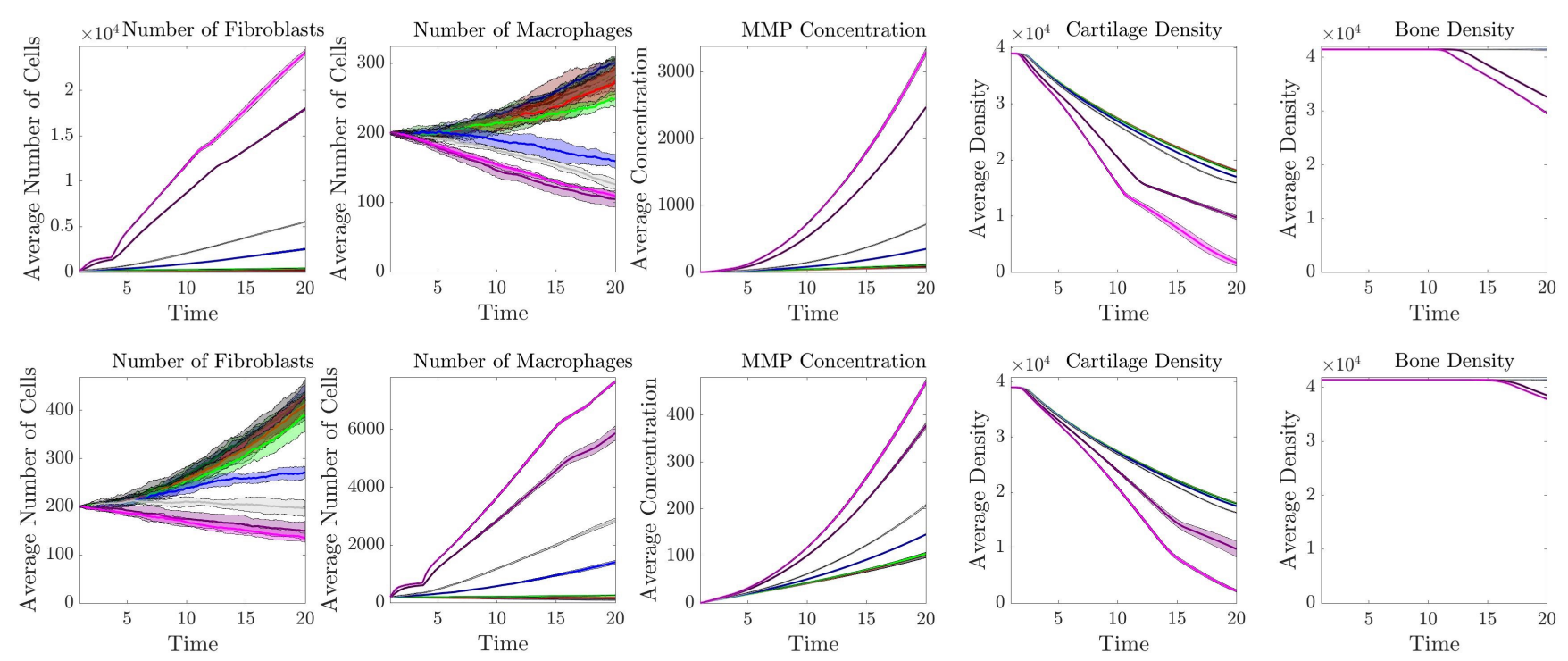

$$
-S_{1}=0.001-S_{2}=0.005-S_{3}=0.01-S_{4}=0.05-S_{5}=0.1-S_{6}=0.5-S_{7}=1-S_{8}=5-S_{9}=10-S_{10}=50-S_{11}=100
$$

Figure 10: Sensitivity analyses of the division probability of fibroblasts $\alpha_{F}$, (top row), or the division probability of macrophages $\alpha_{M}$, (bottom row). The five columns represent the five key outputs of the model: the total number of fibroblasts over time, the total number of macrophages over time, the global MMP concentration over time, the global cartilage density over time and the global bone density over time. In each subplot the results for each scalar value of $S_{n}$ is plotted as indicated by the legend, where the original values of $\alpha_{F}$ or $\alpha_{M}$ are multiplied by $S_{n}$ in each case. The solid line is the average of five runs of the simulation and the shaded area in the same colour is the standard deviation between runs.

We next consider the probability of cell division for fibroblasts and macrophages, $\alpha_{F}$ and $\alpha_{M}$, respectively, and each sensitivity analysis result is displayed in Figure 10 . For the cases where we vary $\alpha_{F}$ (top row), for the values $S_{n} \leq S_{7}=1$ there is no significant difference in all 5 outputs. For the other values, $S_{n} \geq S_{8}=5$, we observe an increase in the total number of fibroblasts, an increase in the global MMP concentration and a decrease in the total number of macrophages as we increase $\alpha_{F}$. For the larger values of $S_{n} \geq S_{10}=50$, we additionally observe a significant increase in the cartilage and bone degradation levels as we increase fibroblast cell division. Similarly, for the cases where we vary $\alpha_{M}$ (bottom row), for the values $S_{n} \leq S_{7}=1$ there is no significant difference in all 5 outputs. When $S_{n} \geq S_{8}=5$ we observe a decrease in the number of fibroblasts, an increase in the total number of macrophages and an increase in MMP global concentration as we increase $\alpha_{M}$. For the larger values of $S_{n} \geq S_{10}=50$, we additionally observe a significant increase in the cartilage and bone degradation levels as we increase fibroblast cell division. Once again, in both cases, for varying $\alpha_{F}$ or $\alpha_{M}$, the standard deviation between runs is relatively low. 
The results of these sensitivity analyses highlight that the values of $\alpha_{F}$ and, to a lesser extent, $\alpha_{M}$ can significantly alter the output in terms of cartilage and bone degradation. The results also highlight the competition for space between the two cell populations, where an increase in the number of fibroblasts leads to a decrease in the number of macrophages, and vice versa, in some of the parameter settings shown.
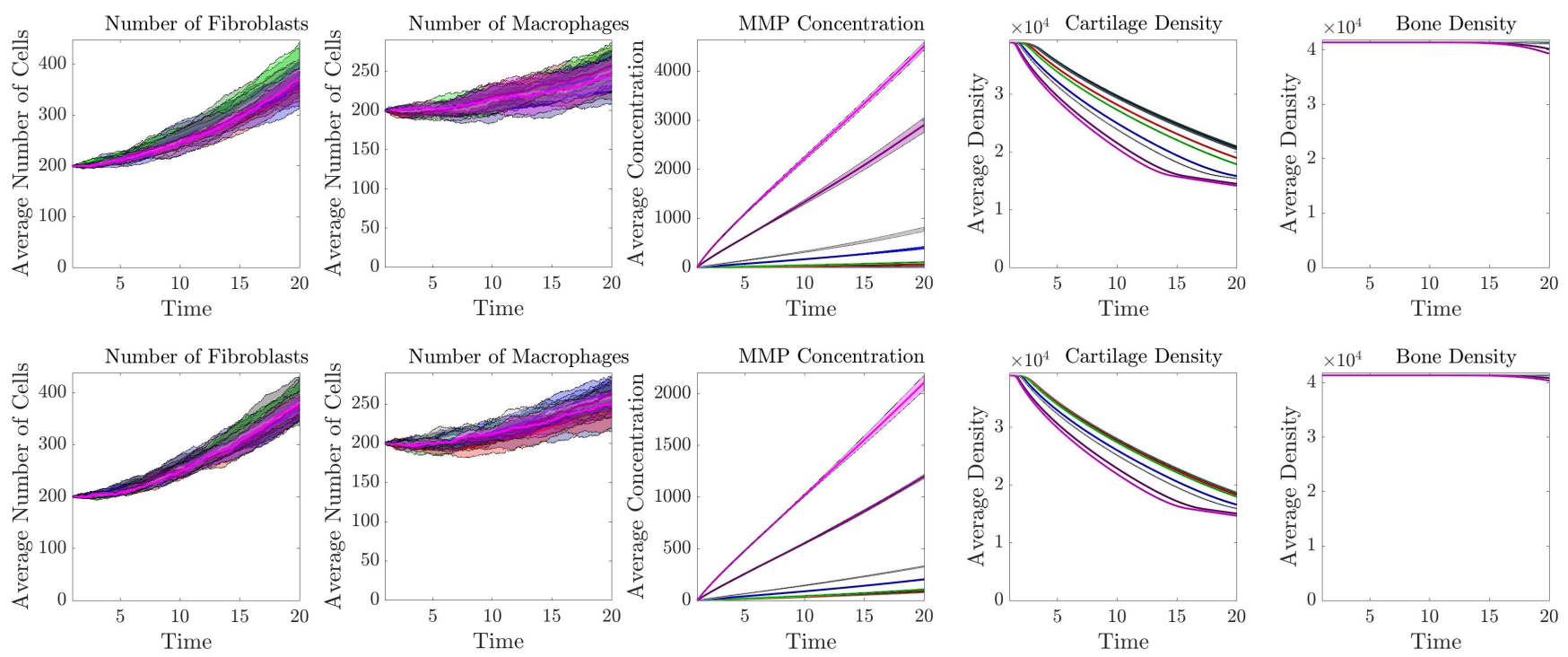

$$
-S_{1}=0.001-S_{2}=0.005-S_{3}=0.01-S_{4}=0.05-S_{5}=0.1-S_{6}=0.5-S_{7}=1-S_{8}=5-S_{9}=10-S_{10}=50-S_{11}=100
$$

Figure 11: Sensitivity analyses of the MMP secretion rate of fibroblasts $\beta_{F}$, (top row), or the MMP secretion rate of macrophages $\beta_{M}$, (bottom row). The five columns represent the five key outputs of the model: the total number of fibroblasts over time, the total number of macrophages over time, the global MMP concentration over time, the global cartilage density over time and the global bone density over time. In each subplot the results for each scalar value of $S_{n}$ is plotted as indicated by the legend, where the original values of $\beta_{F}$ or $\beta_{M}$ are multiplied by $S_{n}$ in each case. The solid line is the average of five runs of the simulation and the shaded area in the same colour is the standard deviation between runs.

In Figure 11, we display the results of sensitivity analyses for varying the values of the MMP secretion rates of both fibroblasts and macrophages, $\beta_{F}$ and $\beta_{M}$. For all values of $S_{n}$ the total number of fibroblasts and macrophages is consistent, as expected, as both macrophage fibroblast movement and proliferation is unaffected by $\beta_{F}$ and $\beta_{M}$. When varying $\beta_{F}$ (top row), for the values of $S_{n} \leq S_{7}=1$ there is no significant change in the global MMP concentration levels, however we observe an increase in these levels once $S_{n} \geq S_{8}=5$. Furthermore, as we increase $\beta_{F}$, for values $S_{n} \geq S_{5}=0.1$, there is an increase in the degradation of cartilage. There is no change in the bone degradation levels, until $S_{n} \geq S_{10}=50$ where there is a slight increase in bone degradation. In the case where we vary $\beta_{M}$ only (bottom row), for $S_{n} \geq 5$ we observe that the global MMP concentration increases as we increase the value of $\beta_{M}$, and the cartilage density decreases over time faster as we increase $\beta_{F}$ for values $S_{n} \geq S_{5}=0.1$. There is additionally a small increase in bone degradation as we increase $\beta_{M}$ for values of $S_{n} \geq S_{10}=50$. Once again, in both cases, for varying $\beta_{F}$ or $\beta_{M}$, the standard deviation between runs is relatively low. The results of these sensitivity analyses highlight that the values of $\beta_{F}$ and $\beta_{M}$ can significantly alter the output in terms of cartilage degradation, but not bone degradation significantly over the time-frame considered. The results also highlight that increasing the secretion rate of fibroblasts seems to have a larger effect on cartilage degradation than increasing the secretion rate of macrophages, this could be due to the larger size of macrophages and the modelling choice that MMPs are only secreted at the centre of the cell. 

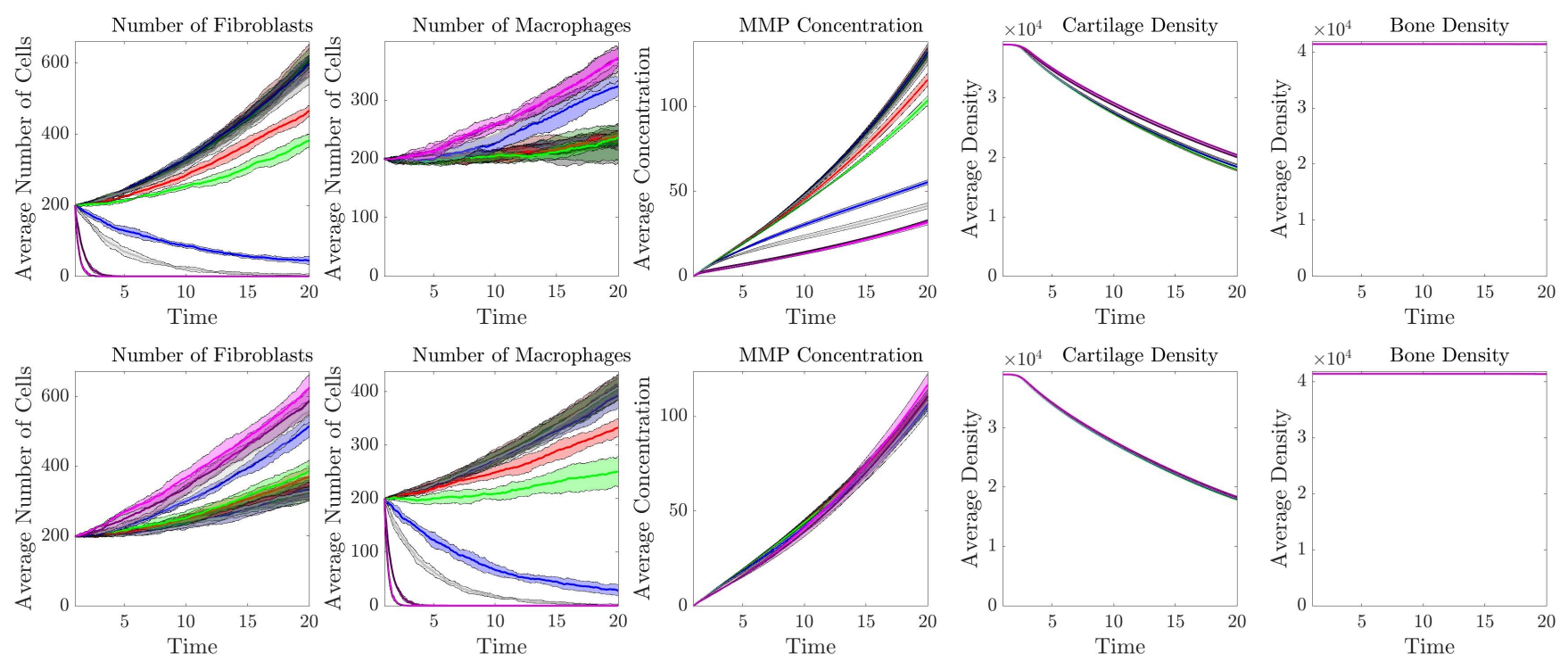

$-S_{1}=0.001-S_{2}=0.005-S_{3}=0.01-S_{4}=0.05-S_{5}=0.1-S_{6}=0.5-S_{7}=1-S_{8}=5-S_{9}=10-S_{10}=50-S_{11}=100$

Figure 12: Sensitivity analyses of the apoptosis probability of fibroblasts $\kappa_{F}$, (top row), or the apoptosis probability of macrophages $\kappa_{M}$, (bottom row). The five columns represent the five key outputs of the model: the total number of fibroblasts over time, the total number of macrophages over time, the global MMP concentration over time, the global cartilage density over time and the global bone density over time. In each subplot the results for each scalar value of $S_{n}$ is plotted as indicated by the legend, where the original values of $\kappa_{F}$ or $\kappa_{M}$ are multiplied by $S_{n}$ in each case. The solid line is the average of five runs of the simulation and the shaded area in the same colour is the standard deviation between runs.

The sensitivity analysis results for varying the apoptosis probabilities of fibroblasts or macrophages, $\kappa_{F}$ and $\kappa_{M}$, are displayed in Figure 12. For the case where we vary $\kappa_{F}$ only (top row), for the values of $S_{n} \leq S_{5}=0.1$ there is no difference in the 5 outputs and for all values of $S_{n}$ there is no change in the bone density levels over the time investigated. For the values of $S_{n} \geq S_{6}=0.5$ we observe a decrease in fibroblasts cell number and a decrease in MMP concentration levels as we increase $\kappa_{F}$. For the values of $S_{n} \geq S_{8}=5$ there appears to be an increase in the total number of macrophages and a small decrease in cartilage degradation as we increase $\kappa_{F}$. When considering the sensitivity analysis for varying $\kappa_{M}$ (bottom row), we observe that for all values of $S_{n}$ there is no significant difference in the global MMP concentration, cartilage density or bone density outputs. For values of $S_{n} \geq S_{8}=5$ there is an increase in the total number of fibroblasts as $\kappa_{F}$ increases, while for values of $S_{n} \geq S_{6}=0.5$ the total number of macrophages decreases as $\kappa_{F}$ increases. The results of these sensitivity analyses on $\kappa_{F}$ and $\kappa_{M}$ suggests that the values of $\kappa_{F}$ and $\kappa_{M}$ will not significantly alter the output in terms of cartilage degradation or bone degradation. However, these parameters can play a role in MMP concentration and the total number of fibroblasts and macrophages. As we increase the probability of death of one cell type, this allows the other cell type to proliferate more freely due to the increased free space, as observed in the figures displayed. As with previous sensitivity analyses, the results are robust as we observe small standard deviation between runs for all cases. 

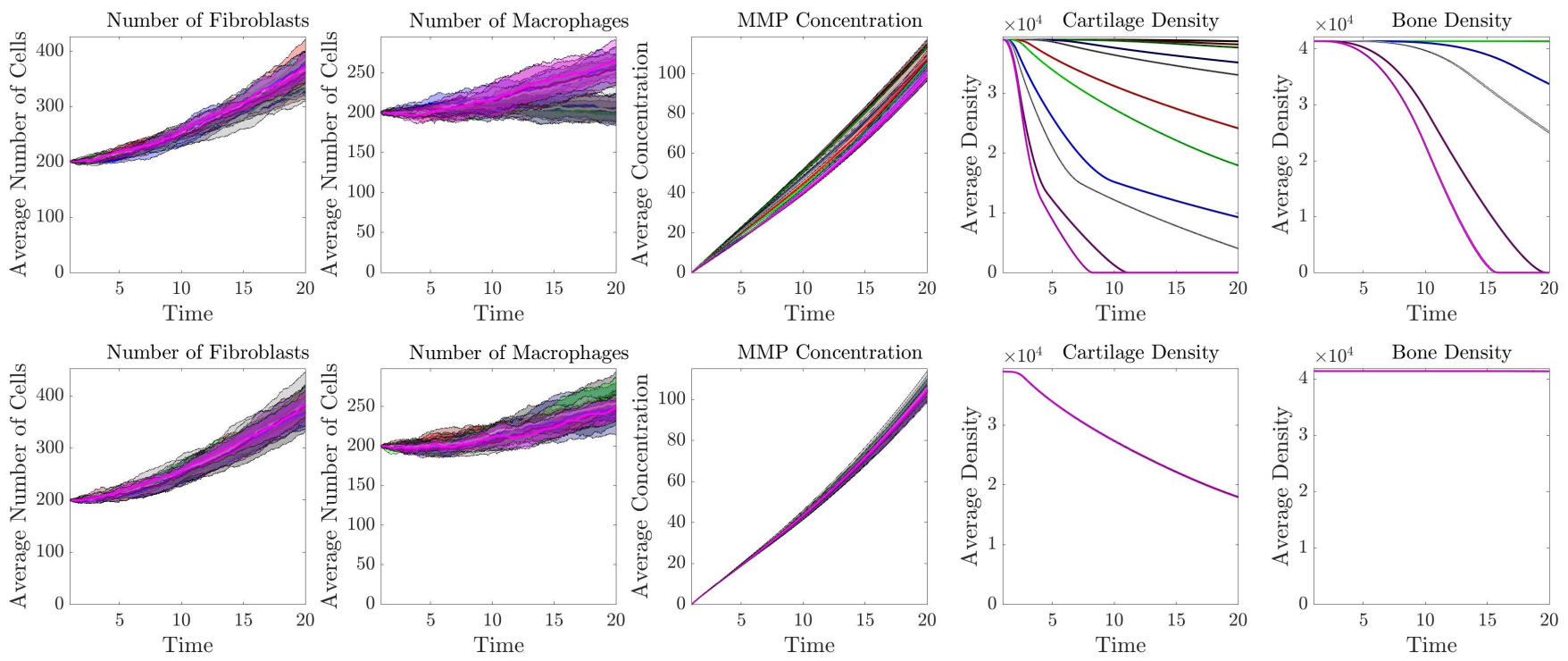

$-S_{1}=0.001-S_{2}=0.005-S_{3}=0.01-S_{4}=0.05-S_{5}=0.1-S_{6}=0.5-S_{7}=1-S_{8}=5-S_{9}=10-S_{10}=50-S_{11}=100$

Figure 13: Sensitivity analyses of the diffusion rate of MMP $\lambda_{C M M P}$, (top row), or the MMP decay rate $\kappa_{C M M P}$, (bottom row). The five columns represent the five key outputs of the model: the total number of fibroblasts over time, the total number of macrophages over time, the global MMP concentration over time, the global cartilage density over time and the global bone density over time. In each subplot the results for each scalar value of $S_{n}$ is plotted as indicated by the legend, where the original value of $\lambda_{C M M P}$ is multiplied by $S_{n}$ in each case. The solid line is the average of five runs of the simulation and the shaded area in the same colour is the standard deviation between runs.

Next we consider the diffusion rate of MMPs, $\lambda_{C M M P}$, and the decay rate of MMPs, $\kappa_{C M M P}$, and perform sensitivity analyses with the results displayed in Figure 13 . When varying $\lambda_{C M M P}$ (top row), for all values of $S_{n}$, there is very little difference in the total number of fibroblasts, macrophages and global MMP concentrations in the model outputs. For the values of $S_{n} \geq S_{4}=0.05$ there is significant increase in the cartilage degradation levels as we increase $\lambda_{C M M P}$, while for values of $S_{n} \geq S_{8}=5$ we additionally observe an increase in bone degradation as $\lambda_{C M M P}$ increases. Considering the sensitivity analysis of the decay rate of MMPs, $\kappa_{C M M P}$ (bottom row), we observe that for all values of $S_{n}$ there is no significant change in the 5 model outputs. These results suggest that $\kappa_{C M M P}$ not a key parameter in determining the model results, while varying $\lambda_{C M M P}$ can significantly impact the output in terms of cartilage degradation or bone degradation. As we increase the diffusion rate of the MMPs, this allows the concentration in the cartilage and bone space significantly faster, promoting increased degradation. As with previous sensitivity analyses, the results are robust as we observe small standard deviation between runs for all case 

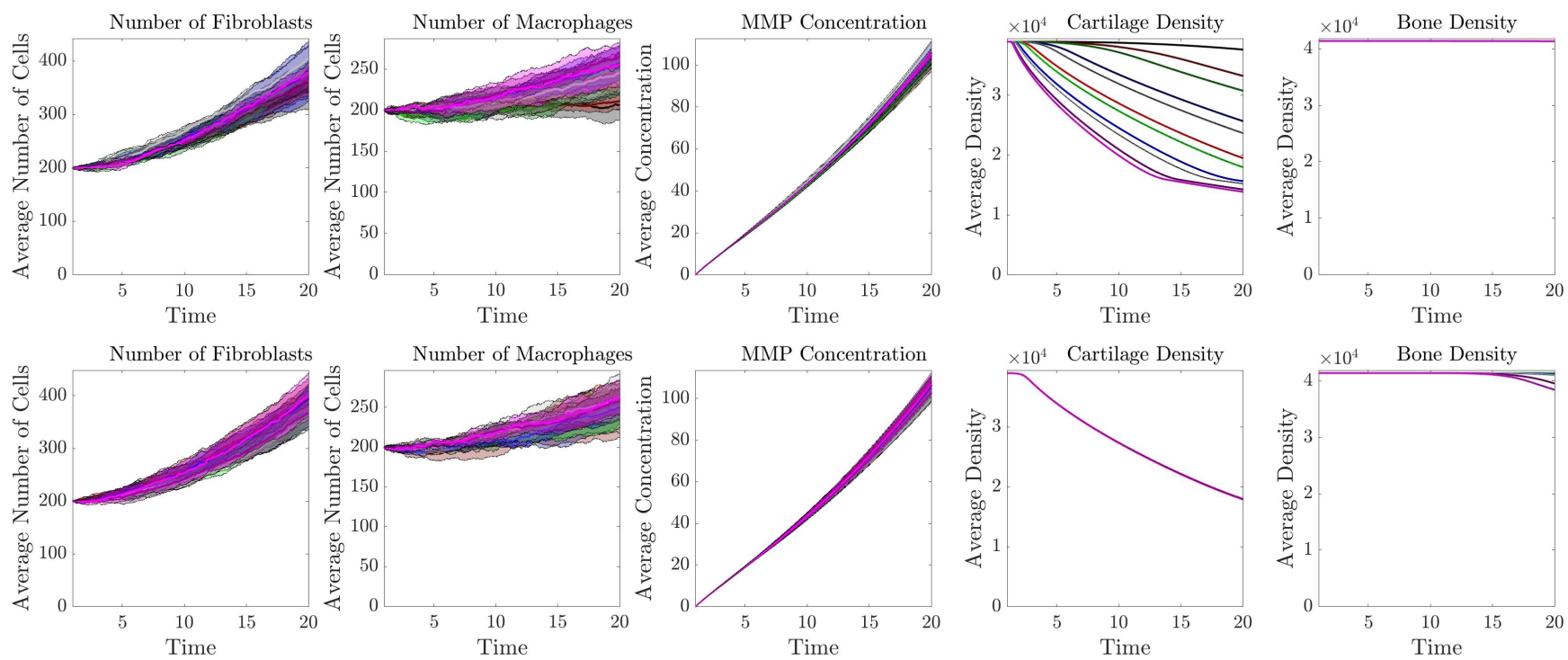

$-S_{1}=0.001-S_{2}=0.005-S_{3}=0.01-S_{4}=0.05-S_{5}=0.1-S_{6}=0.5-S_{7}=1-S_{8}=5-S_{9}=10-S_{10}=50-S_{11}=100$

Figure 14: Sensitivity analyses of the MMP degradation rate of cartilage $\kappa_{\rho_{C}}$, (top row), or the MMP degradation rate of bone $\kappa_{\rho_{B}}$, (bottom row). The five columns represent the five key outputs of the model: the total number of fibroblasts over time, the total number of macrophages over time, the global MMP concentration over time, the global cartilage density over time and the global bone density over time. In each subplot the results for each scalar value of $S_{n}$ is plotted as indicated by the legend, where the original values of $\kappa_{\rho_{C}}$ or $\kappa_{\rho_{B}}$ are multiplied by $S_{n}$ in each case. The solid line is the average of five runs of the simulation and the shaded area in the same colour is the standard deviation between runs.

Finally, we perform sensitivity analyses on the degradation rates of cartilage and bone by MMPs, $\kappa_{\rho_{C}}$ and $\kappa_{\rho_{B}}$, the results of which are displayed in Figure 14. From the results of the sensitivity analysis on $\kappa_{\rho_{C}}$ (top row) we observe that for all values of $S_{n}$ there are no significant differences in the total number of fibroblasts, the total number of macrophages, the MMP concentrations or bone density levels as the values of $\kappa_{\rho_{C}}$ are varied. However, for all values of $S_{n}$ we observe an increase in cartilage degradation as we increase $\kappa_{\rho_{C}}$. From the results of the sensitivity analysis on $\kappa_{\rho_{B}}$ (bottom row) we observe that for all values of $S_{n}$ there are no significant differences in the total number of fibroblasts, the total number of macrophages, the MMP concentrations or cartilage density levels as the values of $\kappa_{\rho_{B}}$ are varied. However, for values of $S_{n} \geq S_{10}=50$ we observe a very slight increase in bone degradation as we increase $\kappa_{\rho_{B}}$. These results suggest that $\kappa_{\rho_{C}}$ and $\kappa_{\rho_{B}}$ only affect the output levels of cartilage and bone, respectively, and not the other outputs of the model. Furthermore, varying $\kappa_{\rho_{C}}$ has a larger effect on affected outputs than $\kappa_{\rho_{B}}$, for the time-frame considered. As with previous sensitivity analyses, the results are robust as we observe small standard deviation between runs for all case

\subsection{Relating our results to RA treatments}

The example results of our model can be related to several therapies currently used to treat rheumatoid arthritis. We can consider drugs that would target or alter the mechanisms described by our model. An overview of the drugs we could consider and the mechanisms that they promote or inhibit are given in Figure 15. 


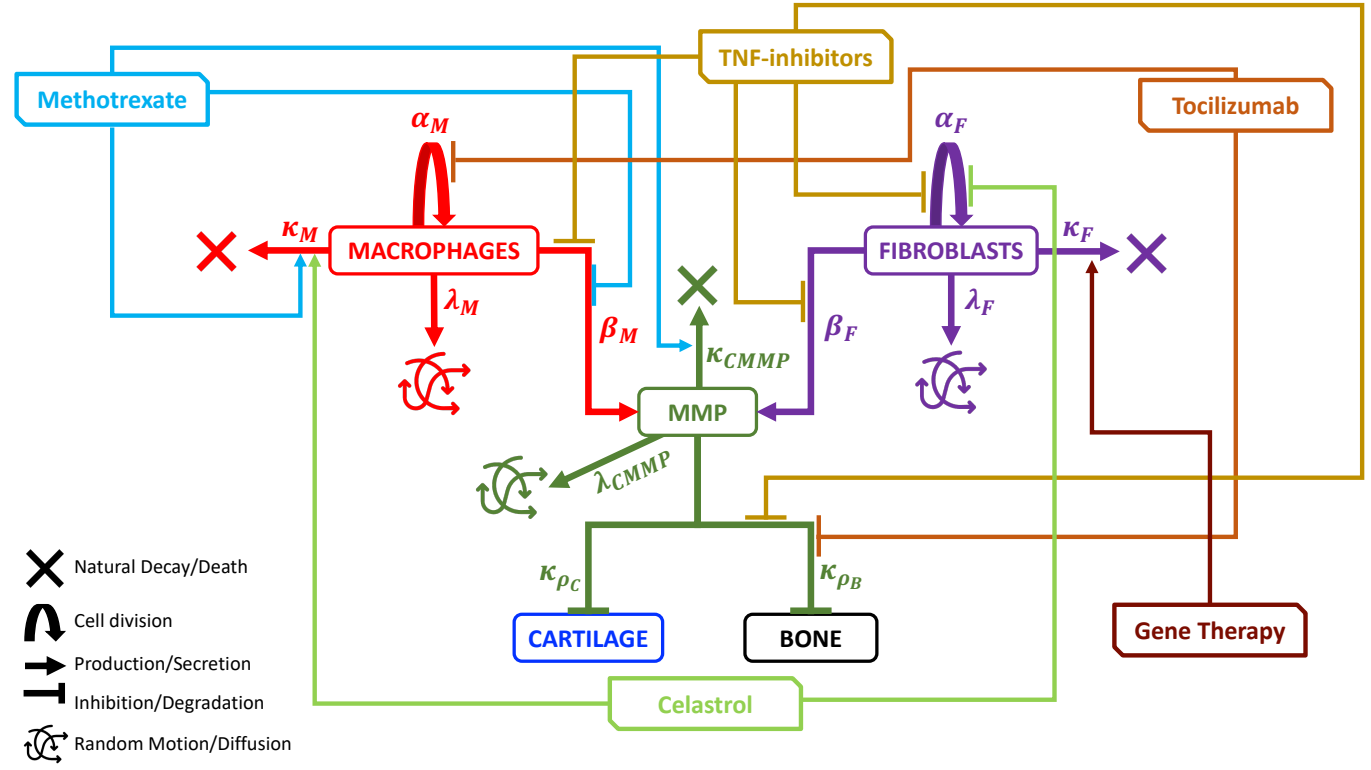

Figure 15: Schematic describing the mechanisms and parameters included in the hybrid model (Figure 4) with potential therapy affects included. TNF- $\alpha$ inhibitors (Gold) can inhibit fibroblast division, inhibit MMP secretion by both macrophages and fibroblasts and inhibit bone degradation in the RA setting. Tocilizumab (Orange) can inhibit macrophage cell division and inhibit bone degradation in the RA setting. Methotrexate (Pale Blue) can promote macrophage apoptosis, promote MMP decay and inhibit MMP secretion by macrophages in the RA setting. Gene therapy (Dark Red) can promote fibroblast apoptosis and celastrol (Pale green) can promote macrophage apoptosis and inhibit fibroblast division in the RA setting.

\subsubsection{Inhibiting immune cell division}

TNF- $\alpha$ inhibitors, such as infliximab, adalimumab and etanercept are commonly prescribed biological disease modifying anti-rheumatic drugs (DMARDs) that target tumour necrosis factor- $\alpha$ (TNF- $\alpha$ ). This cytokine is involved in multiple processes within pannus formation in RA, one of these being the promotion of fibroblast cell division [5, 12, 42. Furthermore, celastrol, an anti-inflammatory chemical has been shown to reduce proliferation rates of fibroblast-like synoviocytes in RA patients 43. Implicitly, we can relate this to a reduction in the probability of fibroblast cell division, $\alpha_{F}$, in our model. By doing this we could expect results similar to those shown in Figure 10 , for the lower values of $S_{n}$, i.e. $S_{n}<S_{7}=1$. We could also explicitly include these drugs, by including the source, diffusion and decay of the drugs across the spatial domain and include the cytokine TNF- $\alpha$ and its mechanisms within the model. As an example, we can consider the effects of the drug celastrol, in 43 it was found that when celastrol is added the fibroblast proliferation rate changed from 0.33 day $^{-1}$ to 0.27 day $^{-1}$. We can replicate this in our model by setting $\alpha_{F}=0.27 \Delta_{\text {tcells }}$, we show the results in Figure 16. From the figure we observe that this small reduction in $\alpha_{F}$ does lead to less fibroblasts in the joint, and less MMPs secreted over time. However, this change is not enough to modify the cartilage degradation levels over the time-frame considered. Therefore these results suggest that using celastrol alone, will be beneficial in reducing immune cell number, but not the overall disease outcomes and further treatments may be required to be used in conjunction with this. 

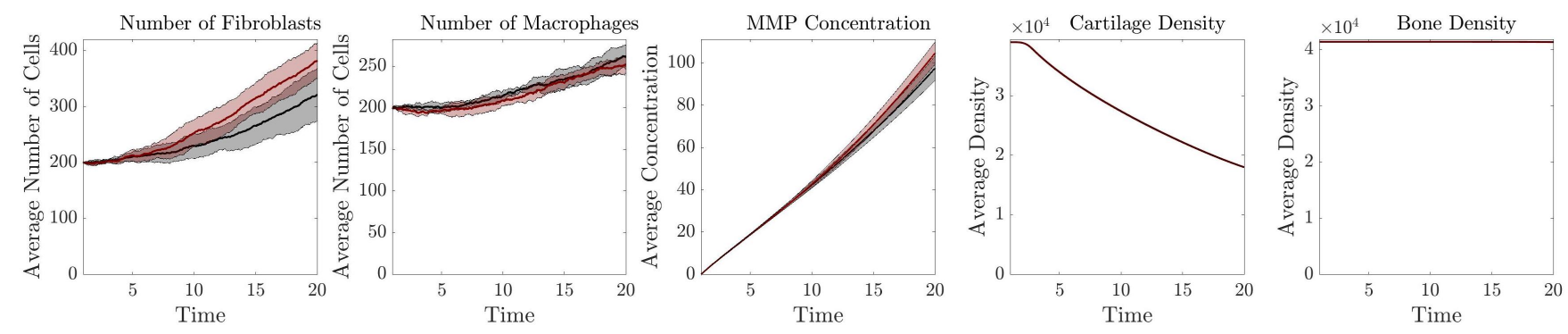

$-\alpha_{F}=0.27 \Delta_{\text {tcells }} \quad-\alpha_{F}=0.33 \Delta_{\text {tcells }}$

Figure 16: Comparing the key outputs of the model for $\alpha_{F}=0.27 \Delta_{\text {tcells }}$ (black) to replicate a setting where the drug celastrol is used, compared to the original results for $\alpha_{F}=0.33 \Delta_{\text {tcells }}$ (red). The five panels represent the five key outputs of the model: the total number of fibroblasts over time, the total number of macrophages over time, the global MMP concentration over time, the global cartilage density over time and the global bone density over time. The solid line is the average of five runs of the simulation and the shaded area in the same colour is the standard deviation between runs.

Tocilizumab is a biological DMARD which targets and inhibits the IL-6 receptor which is produced by several immune cell types. Tocilizumab has been shown to inhibit the cell division of macrophages in the RA setting [42, 44]. Implicitly, we can relate this to a reduction in the probability of macrophage cell division, $\alpha_{M}$, in our model. By doing this we could expect results similar to those shown in Figure 10 , for the lower values of $S_{n}$, i.e. $S_{n}<S_{7}=1$. We could also explicitly include the drug, by including the source, diffusion and decay of the drug across the spatial domain.

\subsubsection{Inhibiting secretion of MMPs by immune cells}

TNF- $\alpha$ inhibitors, have also been shown to inhibit the secretion of MMPs by fibroblasts and macrophages [5, 12, 18]. Implicitly, we can relate this to a reduction in the parameters $\beta_{F}$ and $\beta_{M}$, in our model. By doing this we could expect results similar to those shown in Figure 11, for the lower values of $S_{n}$, i.e. $S_{n}<S_{7}=1$. We could also explicitly include these drugs, by including the source, diffusion and decay of the drugs across the spatial domain and include the cytokine TNF- $\alpha$ and its mechanisms within the model.

Methotrexate is generally the first drug prescribed to treat RA and is efficient in reducing symptoms of the disease such as joint damage and joint pain [5, 45. Methotrexate, which is a conventional synthetic DMARD, has been shown to inhibit the secretion of MMPs by macrophages 42,45. Implicitly, we can relate this to a reduction in the parameter $\beta_{M}$, in our model. By doing this we could expect results similar to those shown in Figure 11, for the lower values of $S_{n}$, i.e. $S_{n}<S_{7}=1$. We could also explicitly include these drugs, by including the source, diffusion and decay of the drugs across the spatial domain.

\subsubsection{Promoting immune cell apoptosis}

Apoptosis of fibroblasts can be induced by targeting specific cell surface receptors [46, for example, genes can be delivered that induce apoptosis in RA fibroblasts, such as with the intra-articular delivery of vectors containing PUMA, a down-stream effector of p53 and an effective inducer of apoptosis [47, 48]. Implicitly, we can relate this to an increase in the parameter $\kappa_{F}$, in our model. By doing this we could expect results similar to those shown in Figure 12, for the higher values of $S_{n}$, i.e. $S_{n}>S_{7}=1$. We could also explicitly include this therapy by considering the mechanisms in further detail.

It has been shown that the drugs methotrexate [42, 45] and chemotherapy through celastrol [49] can increase the apoptosis of macrophages within the RA setting. Implicitly, we can relate these to an increase in the parameter $\kappa_{M}$, in our model. By doing this we could expect results similar to those shown in Figure 12, for the higher values of $S_{n}$, i.e. $S_{n}>S_{7}=1$. We could also explicitly include these drugs by including the source, diffusion and decay of the drugs across the spatial domain.

\subsubsection{Promoting MMP decay}

Methotrexate has also been shown to directly increase the decay of MMPs in the RA setting, by increasing the levels 
of tissue inhibitors of metalloproteinases (TIMPs) 5]. Implicitly, this relates to an increase in the parameter $\kappa_{C M} M P$ in our model. By doing this we could expect results similar to those shown in Figure 13 , for the higher values of $S_{n}$, i.e. $S_{n}>S_{7}=1$. Again, we could explicitly include the mechanisms of methotrexate and MMPs for a more detailed description.

\subsubsection{Inhibiting bone erosion}

Bone erosion is facilitated through osteoclasts which are activated through MMPs. Tocilizumab and TNF-inhibitors have both been shown to inhibit the activity of osteoclasts and therefore reduce bone degradation [5, 9, 12, 18]. Implicitly, in our model we could relates this to a reduction in the parameter $\kappa_{\rho_{B}}$, and by doing this we would expect results similar to those shown in Figure 14 for the lower values of $S_{n}$, i.e. $S_{n}<S_{7}=1$. Again, we could explicitly include these drugs and the osteoclasts within the bone for a more detailed description.

\section{Discussion and conclusions}

In this study we introduced a new stochastic hybrid model to describe the growth and development of the pannus within a small proximal interphalangeal (PIP) joint, in the context of rheumatoid arthritis (RA). The model combined an individual-based approach for cell dynamics with a continuum approach for MMPs, cartilage and bone dynamics. We first provided some initial results that considered three scenarios with increasing proliferation rates of cells to highlight different contexts of rheumatoid arthritis (Figures 5.77). These results further highlighted that even when we observed spatial heterogeneity between simulation results (Figure 8), we still obtain low standard deviation in the global MMP concentrations, global cartilage density and global bone density outputs. This result highlighted that observing varied spatial patterns in the pannus, can still result in the same outcomes and rate of degradation in the joint.

Performing sensitivity analyses on the hybrid model gave some insight into which parameters play a key role in determining the outcome of the model. The results highlighted that varying the immune cell movement probabilities (Figure 9), varying the apoptosis rates of cells (Figure 12), varying the decay rate of MMPs (Figure 13) or varying the degradation rate of bone by MMPs (Figure 14) did not significantly affect cartilage and bone degradation. On the other hand, the results showed that bone and cartilage density can be significantly reduced when we increase the division probability of cells (Figure 10), the MMP secretion rates of cell (Figure 11), the diffusion rate of MMPs (Figure 13) or the degradation rates of cartilage (Figure 14). Overall, these sensitivity results identify the parameters that cause the greatest variations in cartilage or bone density degradation levels. Further experimental and clinical studies will have to be performed to estimate better these parameter values.

Finally in Section 3.3, we discussed the above numerical results in the context of current therapeutic approaches for RA. In particular, we emphasised the model parameters that could be varied to investigate (implicitly) the effects of different drug therapies, and how these changes in model parameters were captured by our numerical simulations. We note that the specific inclusion of the dynamics of these drugs into the mathematical model, which was not the purpose of this particular study, could lead to more complex dynamics due to potential nonlinear effects between different system components. This will be the focus of a future study.

As described in Section 1.1, there are various layers of biological complexity that have not been included in the model so far. The aim of this study was to present a simple stochastic model that can reproduce the degradation of the cartilage and the bone in the presence of large numbers of fibroblasts and macrophages, that can secrete MMPs. This framework can be easily extended using the current methods to incorporate some of these details. For example, we could incorporate further mechanisms of the fibroblasts or macrophages, such as influx and recruitment of the immune cells from out-with the joint through the vasculature. Furthermore, using the individual-based approach we could incorporate heterogeneity whereby each macrophage or fibroblast exhibits different phenotypes in regards to their probability of movement or division. Further immune cell types could also be included such as T cells and B cells using similar techniques to the inclusion of the macrophages and fibroblasts in the current framework. The cartilage and bone densities could be more explicitly considered by including the cell types within these tissues. Chondrocytes within cartilage that produce the cartilage extra-cellular matrix (ECM) could be included. The (im)balance between osteoclasts and osteoblasts in bone could also be investigated in the RA context further by including these cell types and their mechanisms explicitly. To incorporate these features we could consider method similar to those used to previously model cartilage growth [50] or bone remodelling [51 56. Finally, if including further aspects of pannus formation and RA progression we could consider explicitly including vasculature into the tissue along with the influx of oxygen and nutrients into the system, modelling these chemicals in as similar way to the MMPs in this work. In RA, the growth of the pannus can result in local hypoxia driving angiogenesis which can further promote an influx of pro-inflammatory immune cells entering the joint $[13$. These suggested extensions to the framework would 
rely on the availability of clinical and experimental data to validate the modelling choices and parameter settings investigated.

\section{Acknowledgments}

\section{Conflict of interest}

All authors declare no conflicts of interest in this paper.

\section{References}

[1] National Rheumatoid Arthritis Society, What is RA?, https://www.nras.org.uk/what-is-ra-article, accessed: 2019-11-01.

[2] E. Calabresi, F. Petrelli, A. F. Bonifacio, I. Puxeddu, A. Alunno, One year in review 2018: Pathogenesis of rheumatoid arthritis, Clin. Exp. Rheumatol. 36 (2) (2018) 175-184.

[3] M. S. Chimenti, P. Triggianese, P. Conigliaro, E. Candi, G. Melino, R. Perricone, The interplay between inflammation and metabolism in rheumatoid arthritis, Cell Death Dis. 6 (9) (2015) e1887.

[4] NHS Choices, Rheumatoid arthritis, https://www.nhs.uk/conditions/rheumatoid-arthritis/, accessed: 2019-10-01.

[5] Q. Guo, Y. Wang, D. Xu, J. Nossent, N. J. Pavlos, J. Xu, Rheumatoid arthritis: Pathological mechanisms and modern pharmacologic therapies, Bone Res. 6 (1) (2018) 15.

[6] E. C. Goligher, J. Duryea, M. H. Liang, F. Wolfe, A. Finckh, Radiographic joint space width in the fingers of patients with rheumatoid arthritis of less than one year's duration, Arthritis Rheumatol. 54 (5) (2006) 1440-1443.

[7] M. S. Saltzherr, G. S. R. Muradin, I. K. Haugen, R. W. Selles, J. W. van Neck, J. H. Coert, J. M. W. Hazes, J. J. Luime, Cartilage evaluation in finger joints in healthy controls and early hand osteoarthritis patients using high-resolution MRI, Osteoarthr. Cartil. 27 (8) (2019) 1148-1151.

[8] O. Schenk, Y. Huo, K. L. Vincken, M. A. van de Laar, I. H. H. Kuper, K. C. H. Slump, F. P. J. G. Lafeber, H. B. J. B. Moens, Validation of automatic joint space width measurements in hand radiographs in rheumatoid arthritis, J. Med. Imaging 3 (4) (2016) 044502.

[9] G. S. Firestein, I. B. McInnes, Immunopathogenesis of rheumatoid arthritis, Immunity 46 (2) (2017) 183-196.

[10] L. Ouboussad, A. N. Burska, A. Melville, M. H. Buch, Synovial tissue heterogeneity in rheumatoid arthritis and changes with biologic and targeted synthetic therapies to inform stratified therapy, Front. Med. 6 (2019) 45.

[11] B. Bartok, G. S. Firestein, Fibroblast-like synoviocytes: Key effector cells in rheumatoid arthritis, Immunol. Rev. 233 (1) (2010) 233-255.

[12] P. Castro-Sánchez, P. Roda-Navarro, Role of CD4+ T cells in Rheumatoid Arthritis, Physiology and Pathology of Immunology, INTECH Open, 2017, pp. 149-171.

[13] D. A. Fox, A. Gizinski, R. Morgan, S. K. Lundy, Cell-cell interactions in rheumatoid arthritis synovium, Rheumatic Dis. Clinics 36 (2) (2010) 311-323.

[14] I. A. Udalova, A. Mantovani, M. Feldmann, Macrophage heterogeneity in the context of rheumatoid arthritis, Nat. Rev. Rheum. 12 (8) (2016) 472.

[15] A. P. Croft, A. J. Naylor, J. L. Marshall, D. L. Hardie, B. Zimmermann, J. Turner, G. Desanti, H. Adams, A. Yemm, U. Müller-Ladner, et al., Rheumatoid synovial fibroblasts differentiate into distinct subsets in the presence of cytokines and cartilage, Arthritis Res. Ther. 18 (1) (2016) 1-11.

[16] J. A. Singh, C. Cameron, S. Noorbaloochi, T. Cullis, M. Tucker, R. Christensen, E. Ghogomu, D. Coyle, T. Clifford, P. Tugwell, et al., Risk of serious infection in biological treatment of patients with rheumatoid arthritis: A systematic review and meta-analysis, The Lancet 386 (9990) (2015) 258-265. 
[17] J. L. Nam, K. Takase-Minegishi, S. Ramiro, K. Chatzidionysiou, J. S. Smolen, D. Van Der Heijde, J. W. Bijlsma, G. R. Burmester, M. Dougados, M. Scholte-Voshaar, et al., Efficacy of biological disease-modifying antirheumatic drugs: A systematic literature review informing the 2016 update of the EULAR recommendations for the management of rheumatoid arthritis, Ann. Rheum. Dis. 76 (6) (2017) 1113-1136.

[18] B. Heidari, Rheumatoid arthritis: Early diagnosis and treatment outcomes, Caspian J. Intern. Med. 2 (1) (2011) 161.

[19] S. Sardar, Å. Andersson, Old and new therapeutics for rheumatoid arthritis: In vivo models and drug development, Immunopharmacol. Immunotoxicol. 38 (1) (2016) 2-13.

[20] F. R. Macfarlane, M. A. J. Chaplain, R. Eftimie, Quantitative predictive modelling approaches to understanding rheumatoid arthritis: A brief review, Cells 9 (1) (2020) 74.

[21] M. J. H. de Hair, J. W. G. Jacobs, J. L. M. Schoneveld, J. M. van Laar, Difficult-to-treat rheumatoid arthritis: An area of unmet clinical need, Rheumatology 57 (7) (2017) 1135-1144.

[22] O. I. Eseonu, C. De Bari, Homing of mesenchymal stem cells: Mechanistic or stochastic? Implications for targeted delivery in arthritis, Rheumatology 54 (2) (2015) 210-218.

[23] J. Mucke, A. Hoyer, R. Brinks, E. Bleck, T. Pauly, M. Schneider, S. Vordenbäumen, Inhomogeneity of immune cell composition in the synovial sublining: Linear mixed modelling indicates differences in distribution and spatial decline of CD68+ macrophages in osteoarthritis and rheumatoid arthritis, Arth. Res. Ther. 18 (1) (2016) $1-10$.

[24] C. D. Buckley, C. Ospely, S. Gay, K. S. Midwood, Location, location, location: How the tissue microenvironment affects inflammation in RA, Nat. Rev. Rheum. 17 (4) (2021) 195-212.

[25] K. L. Winthrop, M. E. Weinblatt, J. Bathon, G. R. Burmester, P. J. Mease, L. Crofford, V. Bykerk, M. Dougados, J. T. Rosenbaum, X. Mariette, et al., Unmet need in rheumatology: Reports from the targeted therapies meeting 2019, Annal. Rheum. Dis. 79 (1) (2020) 88-93.

[26] M. G. van de Sande, D. L. Baeten, Immunopathology of synovitis: From histology to molecular pathways, Rheumatology 55 (4) (2016) 599-606.

[27] E. N. Bodine, R. M. Panoff, E. O. Voit, A. E. Weisstein, Agent-based modeling and simulation in mathematics and biology education, Bull, Math. Biol. 82 (8) (2020) 1-19.

[28] P. A. D. M. Van Liedekerke, A. Buttenschön, D. Drasdo, Off-lattice agent-based models for cell and tumor growth: Numerical methods, implementation, and applications, in: Numerical methods and advanced simulation in biomechanics and biological processes, Elsevier, 2018, pp. 245-267.

[29] V. Baldazzi, F. Castiglione, M. Bernaschi, An enhanced agent based model of the immune system response, Cell. Immunol. 244 (2) (2006) 77-79.

[30] C. K. Macnamara, Biomechanical modelling of cancer: Agent-based force-based models of solid tumours within the context of the tumour microenvironment, Comput. Syst. Oncol. 1 (2) (2021) e1018.

[31] M. L. Martins, S. C. Ferreira Jr, M. J. Vilela, Multiscale models for biological systems, Curr. Opin. Colloid Interface Sci. 15 (1-2) (2010) 18-23.

[32] A. M. Bailey, B. C. Thorne, S. M. Peirce, Multi-cell agent-based simulation of the microvasculature to study the dynamics of circulating inflammatory cell trafficking, Ann. Biomed. Eng. 35 (6) (2007) 916-936.

[33] R. C. Cockrell, G. An, Examining the controllability of sepsis using genetic algorithms on an agent-based model of systemic inflammation, PLoS Comp. Biol. 14 (2) (2018) e1005876.

[34] A. Bayani, J. L. Dunster, J. J. Crofts, M. R. Nelson, Spatial considerations in the resolution of inflammation: Elucidating leukocyte interactions via an experimentally-calibrated agent-based model, PLoS Comp. Biol. 16 (11) (2020) e1008413.

[35] J. S. Lowengrub, H. B. Frieboes, F. Jin, Y. L. Chuang, X. Li, P. Macklin, S. M. Wise, V. Cristini, Nonlinear modelling of cancer: Bridging the gap between cells and tumours, Nonlinearity 23 (1) (2009) R1. 
[36] P. Macklin, M. E. Edgerton, Discrete cell modeling, in: V. Cristini, J. S. Lowengrub (Eds.), Multiscale modelling of cancer: An integrated experimental and mathematical modeling approach, Cambridge University Press, 2010, pp. 88-122.

[37] G. G. Powathil, M. Swat, M. A. J. Chaplain, Systems oncology: Towards patient-specific treatment regimes informed by multiscale mathematical modelling, in: Sem. Cancer Biol., Vol. 30, Elsevier, 2015, pp. 13-20.

[38] K. A. Rejniak, A. R. A. Anderson, Hybrid models of tumor growth, WIRES Syst. Biol. Med. 3 (1) (2011) $115-125$.

[39] R. Shields, R. F. Brooks, P. N. Riddle, D. F. Capellaro, D. Delia, Cell size, cell cycle and transition probability in mouse fibroblasts, Cell 15 (2) (1978) 469-474.

[40] S. Hamis, S. Stratiev, G. G. Powathil, Uncertainty and sensitivity analyses methods for agent-based mathematical models: An introductory review, The Physics of Cancer: Research Advances (2021) 1-37.

[41] G. Qian, A. Mahdi, Sensitivity analysis methods in the biomedical sciences, Math. Biosci. 323 (2020) 108306.

[42] N. Moise, A. Friedman, Rheumatoid arthritis - A mathematical model, J. Theor. Biol. 461 (2019) 17-33.

[43] Z. Fang, D. He, B. Yu, F. Liu, J. Zuo, Y. Li, Q. Lin, X. Zhou, Q. Wang, High-throughput study of the effects of celastrol on activated fibroblast-like synoviocytes from patients with rheumatoid arthritis, Genes 8 (9) (2017) 221.

[44] J. A. Singh, S. Beg, M. A. Lopez-Olivo, Tocilizumab for rheumatoid arthritis: A Cochrane systematic review, J Rheumatol. 38 (1) (2011) 10-20.

[45] M. A. Lopez-Olivo, H. R. Siddhanamatha, B. Shea, P. Tugwell, G. A. Wells, M. E. Suarez-Almazor, Methotrexate for treating rheumatoid arthritis, Cochrane Database of Systematic Reviews (6). doi:10.1002/14651858. CD000957.pub2

[46] G. Nygaard, G. S. Firestein, Restoring synovial homeostasis in rheumatoid arthritis by targeting fibroblast-like synoviocytes, Nat. Rev. Rheumatol. 16 (6) (2020) 316-333.

[47] H. Cha, S. Rosengren, D. L. Boyle, G. S. Firestein, PUMA regulation and proapoptotic effects in fibroblast-like synoviocytes, Arthritis Rheum. 54 (2) (2006) 587-592.

[48] S. Hong, H. Marotte, G. Courbon, G. S. Firestein, P. Boulanger, P. Miossec, PUMA gene delivery to synoviocytes reduces inflammation and degeneration of arthritic joints, Nat. Commun. 8 (1) (2017) 1-12.

[49] C. Deng, Q. Zhang, P. He, B. Zhou, K. He, X. Sun, G. Lei, T. Gong, Z. Zhang, Targeted apoptosis of macrophages and osteoclasts in arthritic joints is effective against advanced inflammatory arthritis, Nat. Commun. 12 (1) (2021) 1-15.

[50] D. W. Smith, B. S. Gardiner, J. B. Davidson, A. J. Grodzinsky, Computational model for the analysis of cartilage and cartilage tissue constructs, J. Tissue Eng. Regen. Med. 10 (4) (2016) 334-347.

[51] J. Lammens, M. Maréchal, H. Delport, L. Geris, H. Oppermann, S. Vukicevic, F. P. Luyten, A cell-based combination product for the repair of large bone defects, Bone 138 (2020) 115511.

[52] L. Geris, In silico tools predict effects of drugs on bone remodelling, Nat. Rev. Rheumat. 16 (9) (2020) 475-476.

[53] G. Kerckhofs, J. Sainz, M. Maréchal, M. Wevers, T. Van de Putte, L. Geris, J. Schrooten, Contrast-enhanced nanofocus X-ray computed tomography allows virtual three-dimensional histopathology and morphometric analysis of osteoarthritis in small animal models, Cartilage 5 (1) (2014) 55-65.

[54] A. Carlier, L. Geris, N. van Gastel, G. Carmeliet, H. Van Oosterwyck, Oxygen as a critical determinant of bone fracture healing? A multiscale model, J. Theor. Biol. 365 (2015) 247-264.

[55] C. H. Lo, E. Baratchart, D. Basanta, C. C. Lynch, Computational modeling reveals a key role for polarized myeloid cells in controlling osteoclast activity during bone injury repair, Sci. Rep. 11 (1) (2021) 1-14.

[56] A. Araujo, L. M. Cook, C. C. Lynch, D. Basanta, An integrated computational model of the bone microenvironment in bone-metastatic prostate cancer, Cancer Res. 74 (9) (2014) 2391-2401. 
[57] T. Nakajima, H. Aono, T. Hasunuma, K. Yamamoto, T. Shirai, K. Hirohata, K. Nishioka, Apoptosis and functional Fas antigen in rheumatoid arthritis synoviocytes., Arthritis Rheumat. 38 (4) (1995) 485-491.

[58] P. Italiani, D. Boraschi, From monocytes to M1/M2 macrophages: Phenotypical vs. functional differentiation, Front. Immunol. 5 (2014) 514.

[59] M. E. Young, P. A. Carroad, R. L. Bell, Estimation of diffusion coefficients of proteins, Biotech, Bioeng. 22 (5) (1980) 947-955.

[60] R. A. Freitas, Nanomedicine, Volume I: Basic Capabilities, Vol. 1, Landes Bioscience Georgetown, TX, 1999.

[61] F. Krombach, S. Münzing, A. M. Allmeling, J. T. Gerlach, J. Behr, M. Dörger, Cell size of alveolar macrophages: An interspecies comparison., Environ. Health Perspect. 105 (suppl 5) (1997) 1261-1263.

[62] C. Urbach, N. C. Gordon, I. Strickland, D. Lowne, C. Joberty-Candotti, R. May, A. Herath, D. Hijnen, J. L. Thijs, C. A. Bruijnzeel-Koomen, et al., Combinatorial screening identifies novel promiscuous matrix metalloproteinase activities that lead to inhibition of the therapeutic target IL-13, Chem. Biol. 22 (11) (2015) $1442-1452$.

[63] R. J. LeVeque, Finite difference methods for ordinary and partial differential equations: Steady-state and timedependent problems, SIAM, 2007.

[64] W. Hao, E. D. Crouser, A. Friedman, Mathematical model of sarcoidosis, Proc. Nat. Acad. Sci. 111 (45) (2014) $16065-16070$.

[65] K. Liao, X. Bai, A. Friedman, Mathematical modeling of interleukin-27 induction of anti-tumor T cells response, PLoS One 9 (3) (2014) e91844.

[66] Y. Kim, S. Lawler, M. O. Nowicki, E. A. Chiocca, A. Friedman, A mathematical model for pattern formation of glioma cells outside the tumor spheroid core, J. Theor. Biol. 260 (3) (2009) 359-371. 


\section{A Discretisation of PDEs}

We require an on-lattice approach for numerical simulations of the PDE components of the model, to perform simulation we discretise the PDEs in space and time to allow for this. We consider the lattice to have spatial index positions $i \in\left[1, \ldots, N_{x}\right]$ and $j \in\left[1, \ldots, N_{y}\right]$, and consider the time to be discretised so that the time-step $k=t / \Delta_{\text {tchem }}$ where $\Delta_{t c h e m}$ is some time-step length. We can then discretise the PDE in Equation (2.1) using a fully explicit form to be written as,

$$
c_{M M P_{\mathrm{i}}}^{k+1}=c_{M M P_{\mathrm{i}}}^{k}+\Delta_{t c h e m}\left(\lambda_{C M M P} \mathcal{L}\left(c_{M M P_{\mathrm{i}}}^{k}\right)+\left(\beta_{F} \rho_{F \mathbf{i}}^{k}+\beta_{M} \rho_{M \mathbf{i}}^{k}\right)\left(1-c_{M M P_{\mathrm{i}}}^{k}\right)-\kappa_{C M M P} c_{M M P_{\mathrm{i}}}^{k}\right),
$$

where $c_{M M P_{\mathrm{i}}}^{k}$ is the concentration of MMP at spatial position $\mathbf{i}$ at time-step $k, \Delta_{\text {tchem }}$ is the time-step length and $\mathcal{L}$ is the finite difference Laplacian, such that,

$$
\mathcal{L}\left(c_{M M P_{\mathrm{i}}}^{k}\right)=\frac{c_{M M P_{(i+1, j)}}^{k}+c_{M M P_{(i-1, j)}}^{k}-2 c_{M M P_{(i, j)}}^{k}}{\Delta_{x c h e m}^{2}}+\frac{c_{M M P_{(i, j+1)}}^{k}+c_{M M P_{(i, j-1)}}^{k}-2 c_{M M P_{(i, j)}}^{k}}{\Delta_{y c h e m}^{2}} .
$$

Here, $\Delta_{x c h e m}$ and $\Delta_{y c h e m}$ are the space-step lengths in the $x$ and $y$ direction, respectively. Similarily, the discrete forms of bone and cartilage equations Equations (2.2) and (2.3), can be written as,

$$
\begin{aligned}
& \rho_{C_{\mathbf{i}}}^{k+1}=\rho_{C_{\mathbf{i}}}^{k}-\Delta_{t c h e m} \kappa_{\rho_{C}} \rho_{C_{\mathbf{i}}}^{k} c_{M M P_{\mathbf{i}}}^{k}, \\
& \rho_{B_{\mathbf{i}}}^{k+1}=\rho_{B_{\mathbf{i}}}^{k}-\Delta_{\text {tchem }} \kappa_{\rho_{B}} \rho_{B_{\mathbf{i}}}^{k} c_{M M P_{\mathbf{i}}}^{k},
\end{aligned}
$$

where $\rho_{C_{\mathrm{i}}}^{k}$ and $\rho_{B_{\mathbf{i}}}^{k}$ are the densities of cartilage and bone at spatial position $\mathbf{i}$ at time-step $k$, and $\Delta_{t c h e m}$ is the time-step length.

\section{A.1 Code availability}

The full code used to perform the numerical simulations discussed in this paper is available upon request via email to the corresponding author or can be accessed on GitHub at: https:/github.com/frm3-st-andrews/Arthritis. 


\section{B Parameter estimation}

\begin{tabular}{|c|c|c|}
\hline Symbol & Description & Value \\
\hline$\Delta_{\text {tchem }}$ & time-step length for deterministic components & $1 \times 10^{-4}$ days \\
\hline$\Delta_{t c e l l s}$ & time-step length for stochastic components & $1 \times 10^{-2}$ days \\
\hline$\Delta_{x}$ & grid-step length in $x$ direction & $1 \times 10^{-3} \mathrm{~cm}$ \\
\hline$\Delta_{y}$ & grid-step length in $y$ direction & $1 \times 10^{-3} \mathrm{~cm}$ \\
\hline$W_{B}$ & initial thickness of bone & $0.1 \mathrm{~cm}$ \\
\hline$W_{C}$ & initial thickness of cartilage & $0.04 \mathrm{~cm} 7]$ \\
\hline$W_{J}$ & initial width of joint space & $0.01 \mathrm{~cm} 6,8$ \\
\hline$W_{M}$ & initial width of synovial membrane & $0.004 \mathrm{~cm}[10]$ \\
\hline$W_{S}$ & initial width between edge of domain and the cartilage & $2 W_{M} \mathrm{~cm}$ \\
\hline$x_{l}$ & minimum $x$ value of domain & $0 \mathrm{~cm}$ \\
\hline$x_{h}$ & maximum $x$ value of domain & $0.3 \mathrm{~cm}$ \\
\hline$y_{l}$ & minimum $y$ value of domain & $0 \mathrm{~cm}$ \\
\hline$y_{h}$ & maximum $y$ value of domain & $W_{J}+2 W_{C}+2 W_{B} \mathrm{~cm}$ \\
\hline$\alpha_{F}$ & probability of a fibroblast dividing & $\left.0.33 \Delta_{\text {tcells }} 42,43\right]$ \\
\hline$\alpha_{M}$ & probability of a macrophage dividing & $0.33 \Delta_{\text {tcells }}$ \\
\hline$\beta_{F}$ & concentration of $C M M P$ produced by secreting fibroblasts & $13.912 \times 10^{-5} \Delta_{\text {tcells }} 42$ \\
\hline$\beta_{M}$ & concentration of $C M M P$ produced by secreting macrophages & $5.2717 \times 10^{-5} \Delta_{\text {tcells }} \overline{42}$ \\
\hline$\kappa_{F}$ & probability of a fibroblast undergoing apoptosis & $0.03 \Delta_{\text {tcells }} 42,57$ \\
\hline$\kappa_{M}$ & probability of a macrophage undergoing apoptosis & $0.033 \Delta_{\text {tcells }}[\overline{42}, 58]$ \\
\hline$\lambda_{F}$ & probability of a fibroblast moving via undirected motion & 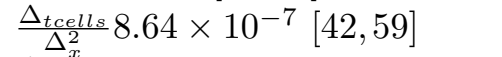 \\
\hline$\lambda_{M}$ & probability of a macrophage moving via undirected motion & $\left.\frac{\Delta_{\text {tocells }}}{\Delta_{r}^{2}} 8.64 \times 10^{-7}, \overline{42,}, \overline{59]}\right]$ \\
\hline$R_{F}$ & Radius of fibroblast & $6.5 \times 10^{-4} \mathrm{~cm} 60$. \\
\hline$R_{M}$ & Radius of macrophage & $10.5 \times 10^{-4} \mathrm{~cm}[61]$ \\
\hline$\lambda_{C M M P}$ & diffusivity of MMPs & $6.59 \times 10^{-2} \mathrm{~cm}^{2}$ day $\left.^{-1} \quad 42,59\right]$ \\
\hline$\kappa_{C M M P}$ & natural decay rate of MMPs & 0.138 day $^{-1}[42,62]$ \\
\hline$\kappa_{\rho_{C}}$ & decay rate of cartilage by MMPs & $\frac{4.44 \times 10^{3}}{1.15} \rho_{C}(0) \mathrm{day}^{-1} 42$ \\
\hline$\kappa_{\rho_{B}}$ & decay rate of bone by MMPs & $0.1 \kappa_{\rho_{C}}$ \\
\hline
\end{tabular}

Table 2: The parameters used in the model. All without references are estimated or calculated. Descriptions of how each value is chosen are provided in $B$.

\section{B.1 Domain parameters}

For the discrete finite-difference equations for the MMPs, the cartilage densities and bone densities we require to set a time-step and grid-spacing for both stochastic and deterministic parts of the model. We choose these to be,

$$
\begin{array}{cl}
\Delta_{\text {tchem }}=1 \times 10^{-4} \text { days, } & \Delta_{\text {tcells }}=1 \times 10^{-2} \text { days, } \\
\Delta_{x}=\Delta_{x c e l l s}=\Delta_{x c h e m}=1 \times 10^{-3} \mathrm{~cm}, & \Delta_{y}=\Delta_{y c e l l s}=\Delta_{y c h e m}=1 \times 10^{-3} \mathrm{~cm} .
\end{array}
$$

These values are chosen to ensure that the finite difference method 63] used to solve the equations are stable for the parameter values chosen. Note that, we choose a different time-step for the deterministic part of the model than the stochastic. This choice is made to speed up the simulations where possible, as a smaller time-step is not required for the stochastic parts. We define the full domain of the grid to be $x \in\left[x_{l}, x_{h}\right]$ and $y \in\left[y_{l}, y_{h}\right]$, where we choose,

$$
x_{l}=y_{l}=0 \mathrm{~cm}, \quad x_{h}=0.3 \mathrm{~cm} \text { and } y_{h}=W_{J}+2 W_{C}+2 W_{B} \mathrm{~cm} .
$$

Therefore, the length of the domain in the $y$ direction depends on the choices of the sub-domain sizes described in Table 1.

\section{B.1.1 Initial height of bone in $y$ directions}

We arbitrarily choose the height of bone in the $y$ direction protruding into the top and bottom of the domain to be,

$$
W_{B}=0.1 \mathrm{~cm} .
$$




\section{B.1.2 Initial width of the cartilage}

In [7, the authors use high resolution MRI (magnetic resonance imaging ) to evaluate the cartilage of the 2nd and 3rd, metacarpophalangeal (MCP) and proximal interphalangeal (PIP) joints in hand osteoarthritis patients and healthy controls. Based in the Netherlands, $41 \mathrm{OA}$ patients and 18 healthy controls were evaluated. The patients were all female, the OA patients were between 40-80 years old, while the healthy individuals were between 18-31 years old. For healthy controls the cartilage thickness in the PIP joint varied between $0.2 \mathrm{~mm}$ and $0.7 \mathrm{~mm}$, with a mean of $0.4 \mathrm{~mm}$ and standard deviation of $0.1 \mathrm{~mm}$. This mean and standard deviation was the same with the OA patients, but they exhibited higher levels of variation. Therefore, we consider that prior to pannus formation, the cartilage width in our simulated PIP joint will be approximately $0.4 \mathrm{~mm}$, as this was the average of healthy controls. Therefore, we set,

$$
W_{C}=0.04 \mathrm{~cm},
$$

where we consider the cartilage to be uniform width surrounding the bone.

\section{B.1.3 Initial width of joint space}

In [6], the authors use radiographs to assess the joint space width (JSW) in undamaged MCP and PIP joints of patients with early rheumatoid arthritis (RA). The mean JSW of the 4 fingers on both hands was measured for the each of the 38 patients that were assessed. The cohort was made up of 29 females and 9 males, 13 patients were under 50 years old, 8 patients were 50-60 years old, and 17 were over 60 years old. In the males the average joint space width was $1.28 \mathrm{~mm}( \pm 0.1 \mathrm{~mm}$ for the $95 \%$ confidence interval $)$, while females were $0.99 \mathrm{~mm}( \pm 0.05 \mathrm{~mm})$. The averages between the age groups were all roughly the same, $(1.1 \mathrm{~mm}, 1.06 \mathrm{~mm}, 1.02 \mathrm{~mm}$, respectively).

Similarly, in 8 the authors use radiographs to validate the joint space width (JSW) calculations in undamaged MCP and PIP joints of patients with early rheumatoid arthritis (RA). Two dutch data sets were used to validate the method, where radiographs were taken every 6 months of the patients. They authors use data from the 4 fingers on each hand, of each patient (omitting the thumbs). For the 1st data set, the average PIP joint in undamaged cases (527 images tested) was $1 \pm 0.2 \mathrm{~mm}$, while in the 2 nd data set (570 images tested) the average was $0.9 \pm 0.2 \mathrm{~mm}$.

Therefore, we consider that prior to pannus formation, in our simulated PIP joint we would expect the initial joint width to be approximated as,

$$
W_{J}=0.01 \mathrm{~cm},
$$

where we assume the spacing to be uniform across the width of the joint.

\section{B.1.4 Initial width of the synovial membrane and space between membrane and cartilage}

In health, the synovial membrane contains relatively few cells consisting of an intimal layer of 1-2 cell thickness and a distinct sublining [10]. Therefore we initially want to allow around 2 layers of cells in the membrane, so we choose

$$
W_{M}=0.004 \mathrm{~cm},
$$

This value is set so that an approximate maximum thickness of two macrophages is possible for the cell membrane. We arbitrarily then choose the space between the cartilage and the edge of the domain in the $x$-direction to be,

$$
W_{S}=2 W_{M} \mathrm{~cm}
$$

\section{B.2 Cell parameters}

\section{B.2.1 Division rates of cells}

In [43, the authors study the effects of celastrol, and anti-inflammatory chemical, on the proliferation rates of fibroblast-like synoviocytes from RA patients. In the untreated case the approximate doubling time of FLSs is 2.1 days. This can be used to estimate a growth rate through the equation for exponential growth, $\Delta_{t c h e m}=\frac{70}{r}$, with $\Delta_{\text {tchem }}$ being the doubling time and $r$ the $\%$ growth rate. Using this we can estimate the FLSs will have a proliferation rate of approximately 0.33 day $^{-1}$, which corresponds to the probability of a fibroblast dividing at any time-step as,

$$
\alpha_{F} \approx 0.33 \Delta_{t c e l l s} .
$$

Note, that the authors in 42 also use a similar value for fibroblast division and estimate that macrophage division will be similar. Therefore, we use in our simulations that,

$$
\alpha_{M}=\alpha_{F}=0.33 \Delta_{t c e l l s}
$$




\section{B.2.2 Chemical secretion rates of cells}

In the mathematical model described in [42, the authors estimate the MMP secretion rates of both fibroblasts and macrophages. For our initial simulations we use these values and set,

$$
\beta_{F}=13.912 \times 10^{-5} \Delta_{\text {tcells }} \quad \text { and } \quad \beta_{M}=5.2717 \times 10^{-5} \Delta_{\text {tcells }} .
$$

\section{B.2.3 Apoptosis rates of cells}

In [57, the authors use experimental methods to detect apoptotic cell death in synovial tissues biopsied from 6 patients with RA, and 3 with $\mathrm{OA}$ as controls. They found that $30 \%$ of the RA fibroblast like-synoviocytes were susceptible to apoptosis. The authors of [42], use this value and the assumption that apoptosis takes $24 \mathrm{hrs}$ to estimate that the apoptosis rate of fibroblast like-synoviocytes is around 0.3 day $^{-1}$. This would lead to the probability of a fibroblast undergoing apoptosis to be,

$$
\kappa_{F}=0.3 \Delta_{\text {tcells }}
$$

This value results in proliferation and decay being very close (similar to a homestatic situation) but leads to extinction due to stochasticity. At the moment we choose a value of $10 \%$ of this, to prevent fibroblast extinction and assume the value would be similar to macrophage apoptosis rates.

In the review paper [58], the authors discuss the key phenotypes of monocytes and macrophages. Specifically, they discuss mouse model results, where Ly6C+ intestinal monocytes that have a half-life of 3 weeks. The authors of [42], use this mouse model value and assume that apoptosis of macrophages occurs at the rate of 0.033 day $^{-1}$. Therefore, we set the probability of a macrophage undergoing apoptosis to be,

$$
\kappa_{M}=0.033 \Delta_{\text {tcells }} .
$$

\section{B.2.4 Movement rates of cells}

In many mathematical modelling works animal cell movement is estimated to be of order $1 \times 10^{-11} \mathrm{~cm}^{2} \mathrm{sec}^{-1} 42$, 596466 . This estimate translates to a diffusion rate of $8.64 \times 10^{-7} \mathrm{~cm}^{2}$ day ${ }^{-1}$, which through scaling corresponds to a probability of moving of $\frac{\Delta_{\text {tchem }}}{\Delta_{\text {xcells }}^{2}} 8.64 \times 10^{-7}$. Therefore, we set for fibroblasts,

$$
\lambda_{F}=\frac{\Delta_{t c e l l s}}{\Delta_{x}^{2}} 8.64 \times 10^{-7},
$$

and similarly for macrophages,

$$
\lambda_{M}=\frac{\Delta_{t c e l l s}}{\Delta_{x}^{2}} 8.64 \times 10^{-7} .
$$

We note that these are just estimates and, as macrophages are larger, they may have a lower movement probability. Also note that $\lambda$ is not the total probability, but contributes to this probability as we also consider a volume exclusion process whereby cells cannot overlap in space with each other or with cartilage or bone. This probability relies on $\Delta_{\text {tchem }}$ and $\Delta_{x c e l l s}=\Delta_{x}$ which have to be chosen such that $\lambda \leq 1$.

\section{B.2.5 Radii of cells}

In 61], the authors show that the average diameter of human alveolar macrophages from the lung of 10 participants was $21.2 \pm 0.3 \mu \mathrm{m}$ in diameter. They also highlight that the diameter of human macrophages is much large than that of other species. Furthermore, in [60] it was shown that the average diameter of a fibroblast is between 10-15 $\mu \mathrm{m}$, while for a macrophage the average diameter can be as large as $20-80 \mu \mathrm{m}$. Therefore, we choose the diameter of a macrophage to be approximately $21 \mu \mathrm{m}$, which results in the radius,

$$
R_{M} \approx \frac{21}{2} \mu \mathrm{m}=10.5 \times 10^{-4} \mathrm{~cm}
$$

Furthermore, we choose the diameter of a fibroblast to be approximately $13 \mu \mathrm{m}$, which results in the radius,

$$
R_{F} \approx \frac{13}{2} \mu \mathrm{m}=6.5 \times 10^{-4} \mathrm{~cm}
$$




\section{B.3 Parameters for MMP mechanisms}

The parameters for the mechanisms of MMPs in the joint are taken to be those used in [42]. They estimate the diffusion rate of MMPs, using [59], to be,

$$
\lambda_{C M M P}=6.59 \times 10^{-2} \mathrm{~cm}^{2} \mathrm{day}^{-1} .
$$

Furthermore, they estimate, using [62, the natural decay rate of MMPs to be,

$$
\kappa_{C M M P}=0.138 \text { day }^{-1} .
$$

In [42, they only consider cartilage degradation, which they estimate to occur at the rate,

$$
\kappa_{\rho_{C}}=\frac{4.44 \times 10^{3}}{1.15} \rho_{C}(0) \mathrm{day}^{-1} .
$$

We make the assumption that the decay rate of bone will be lower, and for simulations, take the value,

$$
\kappa_{\rho_{B}}=\frac{\kappa_{\rho_{C}}}{10}=\frac{4.44 \times 10^{3}}{11.5} \rho_{C}(0) \mathrm{day}^{-1}
$$

